\title{
Factors associated with underrepresented minority physician scientist trainee career choices
}

\author{
Aisha L. Siebert ${ }^{1}$, Shinnyi Chou ${ }^{2}$, Omar Toubat ${ }^{3}$, Alexander J. Adami ${ }^{4} \mathbb{D}$, Hajwa Kim$^{5}$, Dania Daye ${ }^{6^{*}}$ and \\ Jennifer M. Kwan ${ }^{7 *}$
}

\begin{abstract}
Background: Recently, there have been concerted efforts to improve racial and ethnic diversity in the physicianscientist workforce. Identifying factors associated with career choices among those underrepresented in medicine and science is a necessary first step to advance this objective. The aim of the present study was to assess the attitudes and factors associated with academic and research career interests among underrepresented predoctoral physician-scientists.
\end{abstract}

Methods: A cross-sectional 70-question survey was distributed to all predoctoral single degree (MD or DO) and dual degree (MD/PhD or DO/PhD) trainees at 32 medical schools in the United States from 2012 to 2014. Main outcomes included factors important to advancement in academic medicine, intended medical specialty, and future career plans. To test the post-hoc hypothesis of whether trainees from underrepresented groups have differing perceptions of career trajectories and obstacles than their counterparts, we evaluated responses according to self-identified race/ethnic status using Chi-square and Fisher's exact tests. All tests were two-sided and significance level of $<0.05$ was used.

Results: There were a total of 4433 responses representing all predoctoral training stages. The response rate was 27\%. Most respondents were single degree trainees (MD/DO 79\% vs MD/DO-PhD 21\%). Most respondents selfidentified as White (67\%), followed by Multi-racial or Other (14.3\%), Asian or Pacific Islander (10.4\%), Hispanic (6\%), and Black or African American (4.1\%). Desired career sector, career intention, and clinical specialty interest differed across race/ethnic groups. With respect to career selection factors, anticipated non-work related responsibilities during residency were also significantly different between these groups. By multivariable regression analysis, Black or African American trainees were significantly less likely than White trainees to indicate a career in academia (OR $0.496,95 \% \mathrm{Cl} 0.322-0.764$ ) and basic research (OR 0.314, 95\% Cl 0.115-0.857), while Multi-racial or Other trainees were also less likely than White trainees to indicate a career in academia (OR 0.763, 95\% Cl 0.594-0.980).

Conclusions: These data represent the first in-depth survey of career aspirations, perceptions, and interests between demographically underrepresented and non-underrepresented predoctoral physician-scientist trainees. Our results identify key differences between these cohorts, which may guide efforts to improve diversity within the physician-scientist workforce.

\footnotetext{
*Correspondence: deldaye@gmail.com; kwanjen@gmail.com

${ }^{6}$ Massachusetts General Hospital, Boston, USA

${ }^{7}$ Section of Cardiovascular Medicine, Yale University School of Medicine, New Haven, CT 06511, USA

Full list of author information is available at the end of the article
}

(c) The Author(s). 2020 Open Access This article is licensed under a Creative Commons Attribution 4.0 International License, which permits use, sharing, adaptation, distribution and reproduction in any medium or format, as long as you give appropriate credit to the original author(s) and the source, provide a link to the Creative Commons licence, and indicate if changes were made. The images or other third party material in this article are included in the article's Creative Commons licence, unless indicated otherwise in a credit line to the material. If material is not included in the article's Creative Commons licence and your intended use is not permitted by statutory regulation or exceeds the permitted use, you will need to obtain permission directly from the copyright holder. To view a copy of this licence, visit http://creativecommons.org/licenses/by/4.0/. The Creative Commons Public Domain Dedication waiver (http://creativecommons.org/publicdomain/zero/1.0/) applies to the data made available in this article, unless otherwise stated in a credit line to the data. 


\section{Background}

Recently, there have been significant efforts aimed at improving racial and ethnic diversity in physician-scientist training programs and the academic biomedical workforce. Organizations such as the National Institutes of Health (NIH) and the Association of American Medical Colleges (AAMC) have defined underrepresented individuals in medicine (URM) as those who self-identify with racial and ethnic groups that are underrepresented in medicine relative to their prevalence in the general population [1, 2]. In 2014, the National Institutes of Health (NIH) Physician-Scientist Workforce Working Group Report identified the need to improve diversity as a principal recommendation for the NIH and other organizations dedicated to training the next generation of physician-scientists [3]. Their analysis not only demonstrated that the number of $\mathrm{MD} / \mathrm{PhD}$ applicants identifying as African American, Native American, or Hispanic have been stagnant for several years, but also that physician-scientists from these groups have been persistently disproportionately underrepresented among $\mathrm{NIH}$ funded investigators. In particular, it was observed that physician-scientist investigators from these demographic groups comprised only $7 \%$ of the total research project grant applicant pool and $4.7 \%$ of awardees [3].

While there are several potential explanations for this disconcerting trend, factors such as opportunities for mentorship, clinical specialty decisions, and structural or implicit bias have all been implicated. Specifically, previous studies have described that URM faculty self-report experiencing less direct mentorship and greater discrimination by superiors or colleagues [4-7]. In addition, prior analyses have shown that even after controlling for academic productivity, URM faculty remain less likely to be promoted and more likely to hold part-time faculty positions than their non-URM peers, suggesting that there may be residual implicit bias in the academic environment and career advancement process [8-10]. While addressing these issues is crucial for improving success among later stage URM physician-scienists, equally important to the effort of improving diversity in the physician-scientist workforce is understanding the intended career choices and obstacles of URM trainees entering the physician-scientist training pipeline. Despite the clear importance of understanding the perspectives of predoctoral URM physician-scientist trainees, there are currently no nationally representative data focusing on this topic.

The American Physician Scientists Association (APSA) is a national, trainee-led organization that was started with the goal of advocating for training and career development opportunities for future physician-scientists [11]. In this role, APSA had led multiple national cohort surveys assessing career interests and attitudes among physician-scientist trainees $[12,13]$. The primary objective of the present study was to evaluate specialty interest, intended career trajectory, and obstacles in a nationally representative cohort of predoctoral physician-scientist trainees, with a particular focus on understanding the factors associated with academic and research career interests among URM trainees. We hypothesized that URM and non-URM trainees differ in academic and research interests and perceive unique career selection factors and obstacles.

\section{Methods \\ Study design}

This study is a cross-sectional survey of predoctoral trainees enrolled in single degree (MD, DO) or dual degree $(\mathrm{MD} / \mathrm{PhD}, \mathrm{DO} / \mathrm{PhD})$ training programs at academic medical institutions in the United States. The main objective of the study was to examine whether there are racial/ethnic differences in career selection factors, perceived research interest, intended medical specialty, experienced and anticipated obstacles, and future career plans in this cohort. The study protocol was reviewed and exempted by the Institutional Review Board at the University of Illinois at Chicago and the University of Pennsylvania. To ensure unique responses, participants submitted school email addresses, which were later deidentified. MD-PhD trainees were those enrolled in tuition sponsorship by MD-PhD programs. MD-RI status was defined by a self-reported career interest of at least $50 \%$ research among single degree candidates. This minimum research interest was selected because $50 \%$ is often the minimum full time effort commitment required for mentored NIH career development awards [14]. All demographic characteristics, including race and ethnicity, were self-defined by respondents. To assess the generalizability of responses from this study cohort, we compared demographics and clinical specialty interests between survey respondents with those of the Association of American Medical College's (AAMC's) enrollment and graduating medical student databases [15]. To facilitate a comparison of specialty interests, we. categorized specialties into the following groups: primary care/medicine, surgical, acute care, diagnostic, and undecided/other (Supplemental Tables 1 and 2).

\section{Survey tool}

A 70-question survey was previously developed and validated at the University of Illinois at Chicago to assess factors important to influencing MD and MD-PhD student interest in research careers (Supplementary material) [12]. For the present study, the survey was administered to all medical students at 32 nationally representative medical schools from 2012 through 2014. According to the AAMC annual report on the total number of matriculants at each 
of the 32 institutions in the year the survey was first administered, the target population for this survey was 16 , 418 trainees [15]. Three reminder emails were sent throughout this collection period, averaging one reminder every 8 months. Students were targeted by email through student list serves, members of the American Medical Women's Association, as well as through institutional representatives of the American Physician Scientists Association (APSA). Institutions were selected with the intention of balancing geographic distribution, funding status (public vs private), and NIH-funded MSTP program representation. The primary criterion for entry was that the student was enrolled in either the single degree (MD, $\mathrm{DO})$ or dual degree $(\mathrm{MD} / \mathrm{PhD}, \mathrm{DO} / \mathrm{PhD})$ track at one of the 32 targeted institutions at the time of survey completion. Participants consented to join the study on the first page of the survey link and were given the option to remove their consent and withdraw participation at any time during the survey. A more complete analysis of career intentions in the entire cohort comparing differences between single and dual-degree trainees was completed and previously published [13]. Data were collected using an online survey tool (SurveyMonkey, www.surveymonkey.com).

\section{Statistical methods}

The survey questions were developed by physicianscientist trainees with feedback from a survey design team and faculty at the University of Illinois at Chicago. Some questions included in this survey were from previously published instruments for graduate and professional student socialization, with permission from the author [16]. The survey consisted of multiple choice and ordinal scale questions developed specifically to permit exploration of differences in the specific aspirations and experiences reported by various respondent characteristics. Assessment of the face validity of items in this questionnaire was conducted by experts in the field as described in the pilot analysis [12]. Chi-squared tests were used to measure associations between categorical variables. Where data violated minimum expected cell counts, Fisher's exact test was performed. Logistic regression was used to determine the unique influence of each predictor variable on the intention to go into academic medicine, and other outcomes such as basic science research, translational research, and clinical research after residency, controlling for other variables in the model: age, sex, ethnicity, training stage, specialty intention, ability to identify a mentor, how medical school was paid for. All tests were performed using SPSS v16. All tests of significance were 2 -sided and a $p$-value less than 0.05 was considered statistically significant.

\section{Results}

\section{Demographics}

There was a total of 4433 responses, yielding an estimated overall response rate of $27 \%$. Of these, 4033 specified their race/ethnicity and were included in this analysis. There were 2566 (63.6\%) MD trainees, 639 $(15.8 \%)$ single-degree trainees interested in pursuing research intense careers (MD-RI), and 828 (20.5\%) dualdegree $\mathrm{MD} / \mathrm{PhD}$ trainees. While the majority of all respondents were in the first 2 years of medical school (53.5\%), all medical and graduate level training stages were represented (Table 1). The majority of respondents self-identified as being White (71\%), followed by Multiracial/Other (14.3\%), Asian (10.5\%), Hispanic (6.0\%), and Black/African American (4.1\%). A similar demographic trend was observed when stratifying trainees according to training paradigm ( $\mathrm{MD}$ vs $\mathrm{MD}-\mathrm{RI}$ vs $\mathrm{MD} / \mathrm{PhD}$ ) (Table 1). Compared to the 2014 AAMC data on the racial/ethnic identities among medical students, respondents to this survey were more likely to be White (71\% vs $55.7 \%$ ) and Multiracial/Other (14.3\% vs $7.2 \%)$, and less likely to be Asian (10.6\% vs $23.3 \%$ ). The proportion of Black/African American (4.1\% vs 5.3\%) and Hispanic $(6.0 \%$ vs $4.7 \%)$ survey respondents were similar to national estimates provided by the AAMC. A complete list of demographic characteristics is summarized in Table 1.

\section{Familial characteristics and financial considerations}

Familial characteristics varied significantly across racial and ethnic groups. Rates of marriage/partnerships were found to be statistically significantly different among respondents, with Asian trainees most likely to be married/partnered (30.6\%), followed by Hispanic (20.8\%), White (16.8\%), Black/African American (14.8\%), and Multiracial/Other respondents $(13.0 \%)$ (Table 1). The proportion of trainees with children demonstrated a different trend, with Black/African American trainees most likely to have children (6.8\%), followed by White (6.7\%), Hispanic (5.0\%), Multiracial/Other (4.1\%), and Asian trainees (1.0\%) (Table 1).

Racial and ethnic differences were also observed with respect to parental educational attainment and methods for financing medical school (Table 1). The majority of Asian trainees (62.8\%) reported having a father and/or mother with an $\mathrm{MD} / \mathrm{DO}$ or $\mathrm{PhD}$ degree. This was followed by Multiracial/Other (56.8\%), White (37.3\%), Black/African American (33.0\%), and Hispanic (26.0\%) trainees. The most common mechanism of paying for medical school tuition for all racial/ethnic groups was loans. However, the second most frequently selected response differed across these groups, with Asians (24.3\%) and Multiracial/Others (23.3\%) indicating family/partner support, Whites (18.2\%) and Hispanics (15.8\%) 
Table 1 Demographic characteristics of respondents by race/ethnicity

\begin{tabular}{|c|c|c|c|c|c|c|c|c|}
\hline Demographic & $\begin{array}{l}\text { Total } \\
\text { n (\%) }\end{array}$ & $\begin{array}{l}\text { Asian or Pacific } \\
\text { Islander } \\
\text { n (\%) }\end{array}$ & $\begin{array}{l}\text { Black or African } \\
\text { American } \\
\mathrm{n}(\%)\end{array}$ & $\begin{array}{l}\text { Multiracial } \\
\text { or Other } \\
\text { n (\%) }\end{array}$ & $\begin{array}{l}\text { White, } \mathrm{n} \\
\text { (\%) }\end{array}$ & $\begin{array}{l}P \text { - } \\
\text { value }\end{array}$ & $\begin{array}{l}\text { Hispanic } \\
\text { n (\%) }\end{array}$ & $\begin{array}{l}P \text {-value } \\
\text { (Hispanic) }\end{array}$ \\
\hline Training pathway & & & & & & $<.0001$ & & 0.008 \\
\hline MD-RI & $\begin{array}{l}639 \\
(15.84 \%)\end{array}$ & 89 (20.84\%) & $35(20.1 \%)$ & $141(24.4 \%)$ & $374(13.1 \%)$ & & $\begin{array}{l}56 \\
(23.1 \%)\end{array}$ & \\
\hline MD-PHD & $\begin{array}{l}828 \\
(20.5 \%)\end{array}$ & 101 (23.7\%) & $30(18.0 \%)$ & $105(18.2 \%)$ & $592(20.7 \%)$ & & $\begin{array}{l}45 \\
(18.6 \%)\end{array}$ & \\
\hline MD & $\begin{array}{l}2566 \\
(63.63 \%)\end{array}$ & $237(55.5 \%)$ & $102(61.1 \%)$ & $331(57.4 \%)$ & $\begin{array}{l}1869 \\
(66.3 \%)\end{array}$ & & $\begin{array}{l}141 \\
(58.3 \%)\end{array}$ & \\
\hline Total & $\begin{array}{l}4033 \\
(100 \%)\end{array}$ & $427(100 \%)$ & $167(100 \%)$ & $577(100 \%)$ & $\begin{array}{l}2862 \\
(100 \%)\end{array}$ & & $\begin{array}{l}242 \\
(100 \%)\end{array}$ & \\
\hline Gender & 0.0034 & & 0.2158 & & & & & \\
\hline Female & $\begin{array}{l}2269 \\
(56.5 \%)\end{array}$ & $259(61.8 \%)$ & $114(68.7 \%)$ & $309(53.8 \%)$ & $\begin{array}{l}1587 \\
(55.6 \%)\end{array}$ & & $\begin{array}{l}133 \\
(55.4 \%)\end{array}$ & \\
\hline Male & $\begin{array}{l}1736 \\
(43.2 \%)\end{array}$ & 159 (38.0\%) & $52(31.3 \%)$ & $262(45.6 \%)$ & $\begin{array}{l}1263 \\
(44.2 \%)\end{array}$ & & $\begin{array}{l}105 \\
(43.8)\end{array}$ & \\
\hline Other & $\begin{array}{l}10 \\
(0.25 \%)\end{array}$ & $1(0.24 \%)$ & $0(--)$ & $3(0.52 \%)$ & $6(0.21 \%)$ & & $2(0.83 \%)$ & \\
\hline TOTAL & $\begin{array}{l}4015 \\
(100 \%)\end{array}$ & $419(100 \%)$ & $166(100 \%)$ & $574(100 \%)$ & $\begin{array}{l}2856 \\
(71.1 \%)\end{array}$ & & $\begin{array}{l}240 \\
(100 \%)\end{array}$ & \\
\hline Training stage & & & & & & 0.0108 & & 0.1864 \\
\hline Medical School Year 1 & $\begin{array}{l}1131 \\
(28.2 \%)\end{array}$ & $128(30.2 \%)$ & $46(27.7 \%)$ & $179(31.1 \%)$ & 778 (27.3\%) & & $\begin{array}{l}84 \\
(35.0 \%)\end{array}$ & \\
\hline Medical School Year 2 & $\begin{array}{l}1015 \\
(25.3 \%)\end{array}$ & 115 (27.1\%) & $35(21.1 \%)$ & $156(27.1 \%)$ & 709 (24.9\%) & & $\begin{array}{l}65 \\
(27.1 \%)\end{array}$ & \\
\hline Medical School Year 3 & $\begin{array}{l}655 \\
(16.6 \%)\end{array}$ & $65(15.3 \%)$ & $29(17.5 \%)$ & $76(13.2 \%)$ & $485(17.5 \%)$ & & $\begin{array}{l}33 \\
(13.8 \%)\end{array}$ & \\
\hline Medical School Year 4 & $\begin{array}{l}666 \\
(16.6 \%)\end{array}$ & $53(12.5 \% 0$ & $32(19.3 \%)$ & $93(14.4 \%)$ & $498(17.5 \%)$ & & $\begin{array}{l}34 \\
(14.2 \%)\end{array}$ & \\
\hline Graduate School Year 1 & $\begin{array}{l}127 \\
(3.16 \%)\end{array}$ & $8(1.89 \%)$ & $7(4.22 \%)$ & $18(3.13 \%)$ & $94(3.30 \%)$ & & $8(3.3 \%)$ & \\
\hline Graduate School Year 2 & $\begin{array}{l}102 \\
(2.54 \%)\end{array}$ & $12(2.83 \%)$ & $3(1.81 \%)$ & $16(2.78 \%)$ & $71(2.49 \%)$ & & $3(1.3 \%)$ & \\
\hline Graduate School Year 3 & $\begin{array}{l}84 \\
(2.09 \%)\end{array}$ & $13(3.07 \%)$ & $3(1.81 \%)$ & $7(1.22 \% 0$ & $61(2.14 \%)$ & & $1(0.4 \%)$ & \\
\hline Graduate School Year 4 & $\begin{array}{l}85 \\
(2.12 \%)\end{array}$ & $10(2.36 \%)$ & $2(1.20 \%)$ & $12(2.08 \%)$ & $18(0.63 \%)$ & & $3(1.3 \%)$ & \\
\hline Graduate School Year 5 or more & $\begin{array}{l}34 \\
(0.84 \%)\end{array}$ & $4(0.94 \%)$ & $1(0.60 \%)$ & $2(0.35 \%)$ & $27(0.95 \%)$ & & $0(0.0 \%)$ & \\
\hline TOTAL & $\begin{array}{l}4018 \\
(100 \%)\end{array}$ & $424(100 \%)$ & $166(100 \%)$ & $576(100 \%)$ & $\begin{array}{l}2852 \\
(100 \%)\end{array}$ & & $\begin{array}{l}240 \\
(100 \%)\end{array}$ & \\
\hline Marital Status & & & & & & $<.0001$ & & 0.0595 \\
\hline Is married/partnered & $\begin{array}{l}1033 \\
(26.2 \%)\end{array}$ & $855(30.6 \%)$ & $62(14.8 \%)$ & $21(13.0 \%)$ & $95(16.8 \%)$ & & $\begin{array}{l}50 \\
(20.8 \%)\end{array}$ & \\
\hline Is NOT married/partnered & $\begin{array}{l}2911 \\
(73.8 \%)\end{array}$ & $357(85.2 \%)$ & $140(87.0 \%)$ & $472(83.3 \%)$ & $\begin{array}{l}1942 \\
(69.4 \%)\end{array}$ & & $\begin{array}{l}190 \\
(79.2 \%)\end{array}$ & \\
\hline TOTAL & $\begin{array}{l}3944 \\
(100 \%)\end{array}$ & 419 (100\%) & $161(100 \%)$ & $567(100 \%)$ & $2797(100 \%)$ & & $\begin{array}{l}240 \\
(100 \%)\end{array}$ & \\
\hline Parental Status & $<.0001$ & & 0.6096 & & & & & \\
\hline Has a child/children & $\begin{array}{l}225 \\
(5.71 \%)\end{array}$ & $4(0.95 \%)$ & $11(6.79 \%)$ & $23(4.06 \%)$ & $187(6.69 \%)$ & & $12(5.0 \%)$ & \\
\hline Does NOT have a child/children & $\begin{array}{l}3718 \\
(94.3 \%)\end{array}$ & 415 (99.1\%) & $151(93.2 \%)$ & $543(95.9 \%)$ & $\begin{array}{l}2609 \\
(93.3 \%)\end{array}$ & & $\begin{array}{l}229 \\
(95.0 \%)\end{array}$ & \\
\hline
\end{tabular}


Table 1 Demographic characteristics of respondents by race/ethnicity (Continued)

\begin{tabular}{llllllll}
\hline Demographic & $\begin{array}{l}\text { Total } \\
\mathbf{n}(\%)\end{array}$ & $\begin{array}{l}\text { Asian or Pacific } \\
\text { Islander } \\
\mathbf{n}(\%)\end{array}$ & $\begin{array}{l}\text { Black or African } \\
\text { American } \\
\mathbf{n}(\%)\end{array}$ & $\begin{array}{l}\text { Multiracial } \\
\text { or Other } \\
\mathbf{n}(\%)\end{array}$ & $\begin{array}{l}\text { White, } \mathbf{n} \\
(\%)\end{array}$ & $\begin{array}{l}\boldsymbol{P} \text { - } \\
\text { value }\end{array}$ & $\begin{array}{l}\text { Hispanic } \begin{array}{l}\boldsymbol{P} \text {-value } \\
\mathbf{n}(\%)\end{array} \\
(\text { Hispanic) }\end{array}$ \\
\hline TOTAL & 3943 & $419(100 \%)$ & $162(100 \%)$ & $566(100 \%)$ & 2796 & 241 \\
& $(100 \%)$ & & & & $(100 \%)$ & $(100 \%)$
\end{tabular}

Advanced degree of mother ${ }^{1}$

\begin{tabular}{|c|c|c|c|c|c|c|c|c|}
\hline $\mathrm{MD}$ or $\mathrm{DO}$ & $\begin{array}{l}330 \\
(8.18 \%)\end{array}$ & 48 (11.2\%) & 7 (4.19\%) & 77 (13.3\%) & 198 (6.92\%) & $<.0001$ & $\begin{array}{l}13 \\
(5.37 \%)\end{array}$ & 0.1120 \\
\hline DDS & $\begin{array}{l}17 \\
(0.42 \%)\end{array}$ & $2(0.47 \%)$ & $1(0.60 \%)$ & $3(0.52 \%)$ & $11(0.38 \%)$ & 0.9454 & $0(0.00 \%)$ & 0.3016 \\
\hline PhD & $\begin{array}{l}208 \\
(5.16 \%)\end{array}$ & 35 (8.2\%) & 11 (6.59\%) & 34 (5.89\%) & $128(4.47 \%)$ & 0.0069 & 7 (2.89\%) & 0.0981 \\
\hline DVM & $9(0.22 \%)$ & $0(--)$ & $0(--)$ & $0(--)$ & $9(0.31 \%)$ & 0.2969 & $0(--)$ & $0.5791^{a}$ \\
\hline Master's & $\begin{array}{l}998 \\
(24.8 \%)\end{array}$ & $120(28.1 \%)$ & $42(25.2 \%)$ & $143(24.8 \%)$ & 693 (24.2\%) & 0.3864 & $\begin{array}{l}47 \\
(19.4 \%)\end{array}$ & 0.0558 \\
\hline
\end{tabular}

Area of medicine mother works in ${ }^{1}$

$\begin{array}{lllllllll}\text { Academia } & 13 & 2(1.20 \%) & 18(3.12 \%) & 70(2.45 \%) & 103(2.55 \%) & 0.4711 & 2(0.83 \%) & 0.0851 \\ \text { Private practice } & (3.04 \%) & & & & & & & \\ & 158 & 16(3.75 \%) & 3(1.80 \%) & 40(6.93 \%) & 99(3.46 \%) & \mathbf{0 . 0 0 0 5} & 4(1.65 \%) & 0.0657 \\ \text { Consulting } & (3.92 \%) & & & & & & & \\ \text { Industry } & 6(0.15 \%) & 0(-) & 0(-) & (-) & 6.15 \%) & 0.4828 & 1(0.41 \%) & 0.2610^{\mathrm{a}} \\ & 12 & 4(0.94 \%) & 0(-) & 1(0.17 \%) & 7(0.24 \%) & 0.0735 & 1(0.41 \%) & 0.3630^{\mathrm{a}} \\ & (0.30 \%) & & & & & & \end{array}$

Advanced degree of father ${ }^{1}$

\begin{tabular}{|c|c|c|c|c|c|c|c|c|}
\hline $\mathrm{MD}$ or $\mathrm{DO}$ & $\begin{array}{l}669 \\
(16.6 \%)\end{array}$ & $75(17.6 \%)$ & $19(11.4 \%)$ & $111(19.2 \%)$ & $464(16.2 \%)$ & 0.0789 & $\begin{array}{l}33 \\
(13.6 \%)\end{array}$ & 0.2296 \\
\hline DDS & $\begin{array}{l}51 \\
(1.26 \%)\end{array}$ & $5(1.17 \%)$ & $2(1.20 \%)$ & $10(1.73 \%)$ & $34(1.19 \%)$ & 0.7566 & $4(1.65 \%)$ & $0.1743^{\mathrm{a}}$ \\
\hline PhD & $\begin{array}{l}512 \\
(12.7 \%)\end{array}$ & $110(25.8 \%)$ & $18(10.8 \%)$ & $106(18.4 \%)$ & $278(9.71 \%)$ & $<.0001$ & $\begin{array}{l}10 \\
(4.13 \%)\end{array}$ & $<.0001$ \\
\hline$J \mathrm{D}$ & $\begin{array}{l}205 \\
(5.08 \%)\end{array}$ & $2(0.47 \%)$ & $2(1.20 \%)$ & $15(2.60 \%)$ & $186(6.50 \%)$ & $<.0001$ & $9(3.72 \%)$ & 0.3275 \\
\hline DVM & $\begin{array}{l}20 \\
(0.50 \%)\end{array}$ & $0(--)$ & $0(--)$ & $1(0.17 \%)$ & $19(0.66 \%)$ & 0.1210 & $0(--)$ & $0.2965^{a}$ \\
\hline Master's & $\begin{array}{l}952 \\
(23.6 \%)\end{array}$ & $118(27.6 \%)$ & 39 (23.4\%) & $159(27.6 \%)$ & $636(22.2 \%)$ & 0.0078 & $\begin{array}{l}41 \\
(16.9 \%)\end{array}$ & 0.0139 \\
\hline \multicolumn{9}{|l|}{ Area of medicine father works in ${ }^{1}$} \\
\hline Academia & $\begin{array}{l}194 \\
(4.81 \% 0\end{array}$ & $22(5.15 \%)$ & $2(1.20 \%)$ & $37(6.41 \%)$ & $133(4.65 \%)$ & 0.0407 & $\begin{array}{l}10 \\
(4.13 \%)\end{array}$ & 0.6337 \\
\hline Private practice & $\begin{array}{l}414 \\
(10.3 \%)\end{array}$ & $40(9.37 \%)$ & 15 (8.98\%) & $71(12.3 \%)$ & $288(10.1 \%)$ & 0.3332 & $\begin{array}{l}21 \\
(8.68 \%)\end{array}$ & 0.4236 \\
\hline Consulting & $\begin{array}{l}31 \\
(0.77 \%)\end{array}$ & $2(0.47 \%)$ & $0(--)$ & $3(0.52 \%)$ & $26(0.91 \%)$ & 0.3917 & $2(0.83 \%)$ & $0.2796^{\mathrm{a}}$ \\
\hline Industry & $\begin{array}{l}33 \\
(0.82 \%)\end{array}$ & $6(1.41 \%)$ & $0(--)$ & $2(0.35 \% 0$ & $25(0.87 \%)$ & 0.1809 & $1(0.41 \%)$ & $0.2790^{\mathrm{a}}$ \\
\hline $\begin{array}{l}\text { How primarily paid for medical } \\
\text { chool }{ }^{1} \mathrm{n}(\% \text { of } 4072)\end{array}$ & & & & & & $<.0001$ & & $<.0001$ \\
\hline MD-PhD or DO-PhD sponsored & $\begin{array}{l}726 \\
(18.28 \%)\end{array}$ & $91(21.7 \%)$ & $25(15.4 \%)$ & $97(17.0 \%)$ & $513(18.2 \%)$ & & $\begin{array}{l}38 \\
(15.77 \%)\end{array}$ & \\
\hline Scholarships & $\begin{array}{l}378 \\
(9.52 \%)\end{array}$ & $43(10.3 \%)$ & $48(29.6 \%)$ & $68(11.9 \%)$ & $219(7.77 \%)$ & & $\begin{array}{l}34 \\
(14.11 \%)\end{array}$ & \\
\hline Grants & $\begin{array}{l}71 \\
(1.79 \%)\end{array}$ & $13(3.10 \%)$ & $6(3.70 \%)$ & $15(2.63 \%)$ & $37(1.31 \%)$ & & $\begin{array}{l}15 \\
(6.22 \%)\end{array}$ & \\
\hline Loans & 2076 & $163(38.9 \%)$ & $77(47.5 \%)$ & $243(42.6 \%)$ & 1593 & & 135 & \\
\hline
\end{tabular}


Table 1 Demographic characteristics of respondents by race/ethnicity (Continued)

\begin{tabular}{|c|c|c|c|c|c|c|c|c|}
\hline Demographic & $\begin{array}{l}\text { Total } \\
\text { n (\%) }\end{array}$ & $\begin{array}{l}\text { Asian or Pacific } \\
\text { Islander } \\
\text { n (\%) }\end{array}$ & $\begin{array}{l}\text { Black or African } \\
\text { American } \\
\mathrm{n}(\%)\end{array}$ & $\begin{array}{l}\text { Multiracial } \\
\text { or Other } \\
\text { n (\%) }\end{array}$ & $\begin{array}{l}\text { White, n } \\
\text { (\%) }\end{array}$ & $\begin{array}{l}P \text { - } \\
\text { value }\end{array}$ & $\begin{array}{l}\text { Hispanic } \\
\text { n (\%) }\end{array}$ & $\begin{array}{l}P \text {-value } \\
\text { (Hispanic) }\end{array}$ \\
\hline & $(52.3 \%)$ & & & & $(56.5 \%)$ & & $(56.02 \%)$ & \\
\hline National Service & $\begin{array}{l}48 \\
(1.21 \%)\end{array}$ & $1(0.24 \%)$ & $1(0.62 \%)$ & $9(1.58 \%)$ & 37 (1.31\%) & & $3(1.24 \%)$ & \\
\hline Personal Savings & $\begin{array}{l}45 \\
(1.13 \%)\end{array}$ & $6(1.43 \%)$ & $0(--)$ & $5(0.88 \%)$ & $34(1.21 \%)$ & & $2(0.83 \%)$ & \\
\hline Family/partner Support & $\begin{array}{l}619 \\
(15.6 \%)\end{array}$ & $102(24.3 \%)$ & $4(2.47 \%)$ & $133(23.3 \%)$ & $380(13.5 \%)$ & & $\begin{array}{l}14 \\
(5.81 \%)\end{array}$ & \\
\hline Work & $5(0.15 \%)$ & $0(--)$ & $0(--)$ & $0(--)$ & $6(0.15 \%)$ & & $0(--)$ & \\
\hline Other & $2(0.05 \%)$ & $0(--)$ & $1(0.62 \%)$ & $0(--)$ & $1(0.04 \%)$ & & $0(--)$ & \\
\hline
\end{tabular}

${ }^{a}$ Fisher's Exact calculated due to minimum cell count violations

${ }^{1}$ Respondents could select all applicable choices, will not sum to $100 \%$

indicating $\mathrm{MD} / \mathrm{PhD}$ or $\mathrm{DO} / \mathrm{PhD}$ sponsorship, and Black/ African American trainees (29.6\%) indicating scholarships.

\section{Career intentions and specialty interest}

We then compared career sector, career intention, and clinical specialty interests (Table 2). Trainees from all ra$\mathrm{cial} / \mathrm{ethnic}$ groups listed academia as the primary career sector of interest (Asian 49.4\%; Black/African American 40.8\%; Multiracial/Other 50.5\%; White 46.4\%; Hispanic 49.4\%). However, secondary and tertiary options were found to split across these groups. Asian and Black/African American trainees expressed interest in hospitalist (Asian 34.5\%; Black/African American 25.5\%) then private practice careers (Asian 19.4\%; Black/African American 22.9\%), whereas Multiracial/Other, White, and Hispanic trainees preferred private practice (Multiracial/ Other 20.4\%; White 28.6\%; Hispanic 24.6\%) over hospitalist careers (Multiracial/Other 18.8\%; White 16.1\%; Hispanic 19.5\%).

With respect to clinical duties, basic/translational research, and clinical research career intentions, there were racial/ethnic differences in the responses across each of these domains (Table 2). White trainees were most likely to indicate wanting to pursue clinical duties (67.6\%) followed by Hispanic (63.1\%), Multiracial/Other (59.7\%), Asian (58.5\%), and Black/African American (56.5\%) trainees. For basic/translational research, Asian trainees were the most interested in pursuing this career intention (19.1\%) followed by Multiracial/Other (17.9\%), Hispanic (17.0\%), White (15.8\%), and Black/African American (14.3\%) trainees. Trends in clinical research interest differed, with Black/African American trainees expressing the greatest interest in this career practice (8.4\%) followed by Hispanic (6.8\%), Multiracial/Other (6.8\%), Asian (6.3\%), and White (4.5\%) trainees. Black/ African American trainees were also most likely to express interest in education and advocacy (Table 2).
Primary care (internal medicine, pediatrics, and family medicine) ranked among the top specialty intentions for all trainees (Table 2). Between primary care specialties, trainees from all racial/ethnic groups preferred pediatrics over internal medicine and family medicine. With regard to general surgery, Hispanics were the most interested in pursuing this specialty (9.1\%) followed by Black/African American (7.7\%), White (6.2\%), Multiracial/Other (6.2\%), and Asian (5.7\%) trainees. The remaining responses for acute care, diagnostic, medical, and surgical sub-specialties are listed in Table 2. To assess generalizability of these trends in specialty interest, we compared our survey responses to those of trainees surveyed in the 2014 AAMC Graduating Student Questionnaire (Supplementary Table 3). When analyzing specialty interest according to pre-specified specialty categories, both our survey $(p=0.001)$ and that of the AAMC $(p=0.01)$ show that specialty interests differ significantly across racial/ethnic groups (Supplementary Tables 1 and 2).

To assess the association between race/ethnicity and intended career path (i.e. academia, basic research, translational research or clinical research) a logistic regression model was used, adjusting for sex, age, training stage, access to mentors, specialty intention, and how medical school was paid for. Asians were significantly more likely to report interest in an academic career (OR 1.69, $95 \mathrm{CI}$ 1.03-2.78), and intention to do basic research (OR 3.229, 95 CI 1.123-9.28) compared to Black/African American trainees (Table 3). Black/African American trainees were less likely to have career intentions in academia (OR 0.49, 95 CI 0.322-0.764) and basic research (OR 0.314 95 CI 0.115-0.857) compared to their White counterparts (Table 3). Similarly, Multiracial/Other respondents were also less likely to have career intentions in academia (OR 0.763, 95 CI 0.594-0.980) than White trainees (Table 3). There were no significant differences in academic, basic/translation, or clinical research 
Table 2 Career sector, career intentions, and clinical specialty interests

\begin{tabular}{|c|c|c|c|c|c|c|c|c|}
\hline & $\begin{array}{l}\text { Total } \\
\text { n (\%) }\end{array}$ & $\begin{array}{l}\text { Asian or Pacific } \\
\text { Islander } \\
\mathbf{n}(\%)\end{array}$ & $\begin{array}{l}\text { Black or African } \\
\text { American } \\
\mathrm{n}(\%)\end{array}$ & $\begin{array}{l}\text { Multiracial or } \\
\text { Other } \\
\mathrm{n}(\%)\end{array}$ & $\begin{array}{l}\text { White } \\
\text { n (\%) }\end{array}$ & $P$-value & $\begin{array}{l}\text { Hispanic } \\
\text { n (\%) }\end{array}$ & $\begin{array}{l}P \text {-value } \\
\text { (Hispanic) }\end{array}$ \\
\hline Sector $^{1}$ & & & & & & $<.0001$ & & 0.5220 \\
\hline Consulting & $71(1.84 \%)$ & $13(3.15 \%)$ & $3(1.91 \%)$ & $10(1.82 \%)$ & $\begin{array}{l}45 \\
(1.65 \%)\end{array}$ & & 7 (2.97\%) & \\
\hline Academia & $\begin{array}{l}1812 \\
(47.1 \%)\end{array}$ & 204 (49.4\%) & $64(40.8 \%)$ & 277 (50.5\%) & $\begin{array}{l}1267 \\
(46.4 \%)\end{array}$ & & $\begin{array}{l}103 \\
(49.36 \%)\end{array}$ & \\
\hline Industry & $66(1.71 \%)$ & $2(0.48 \%)$ & $3(1.91 \%)$ & $11(2.00 \%)$ & $\begin{array}{l}50 \\
(1.83 \%)\end{array}$ & & $5(2.12 \%)$ & \\
\hline Government & $\begin{array}{l}109 \\
(2.83 \%)\end{array}$ & $5(1.215)$ & $5(3.18 \%)$ & $23(4.19 \%)$ & $\begin{array}{l}76 \\
(2.78 \%)\end{array}$ & & $\begin{array}{l}11 \\
(4.66 \%)\end{array}$ & \\
\hline Private Practice & $\begin{array}{l}1009 \\
(26.2 \%)\end{array}$ & $80(19.4 \%)$ & $36(22.9 \%)$ & $112(20.4 \%)$ & $\begin{array}{l}781 \\
(28.6 \%)\end{array}$ & & $\begin{array}{l}58 \\
(24.58 \%)\end{array}$ & \\
\hline Hospitalist & $\begin{array}{l}683 \\
(17.7 \%)\end{array}$ & $101(24.5 \%)$ & $40(25.5 \%)$ & $103(18.8 \%)$ & $\begin{array}{l}439 \\
(16.1 \%)\end{array}$ & & $\begin{array}{l}46 \\
(19.49 \%)\end{array}$ & \\
\hline Other & $34(0.88 \%)$ & $1(0.24 \%)$ & $3(1.91 \%)$ & $4(0.73 \%)$ & $\begin{array}{l}26 \\
(0.95 \%)\end{array}$ & & $3(1.27 \%)$ & \\
\hline N/A & $20(0.52 \%)$ & $3(0.73 \%)$ & $1(0.64 \%)$ & $1(0.18 \%)$ & $\begin{array}{l}15 \\
(0.55 \%)\end{array}$ & & $1(0.42 \%)$ & \\
\hline Nonprofit & $\begin{array}{l}20 \\
(0.52 \% 0\end{array}$ & $0(--)$ & $1(0.64 \%)$ & $3(0.55 \% 0$ & $\begin{array}{l}16 \\
(0.59 \%)\end{array}$ & & $0(--)$ & \\
\hline Community Hospital & $27(0.70 \%)$ & $4(0.97 \%)$ & $1(0.64 \%)$ & $5(0.91 \%)$ & $\begin{array}{l}17 \\
(0.62 \%)\end{array}$ & & $2(0.85 \%)$ & \\
\hline Career Intention ${ }^{1}$ & & & & & & 0.0005 & & 0.5440 \\
\hline Education & $\begin{array}{l}225 \\
(5.85 \%)\end{array}$ & $24(5.83 \%)$ & $12(7.79 \%)$ & $29(5.33 \%)$ & $\begin{array}{l}160 \\
(5.85 \%)\end{array}$ & & 9 (3.81\%) & \\
\hline Basic research & $\begin{array}{l}197 \\
(5.12 \%)\end{array}$ & $28(6.80 \%)$ & $1(0.65 \%)$ & $26(4.78 \%)$ & $\begin{array}{l}142 \\
(5.19 \%)\end{array}$ & & $\begin{array}{l}15 \\
(6.36 \%)\end{array}$ & \\
\hline Clinical research & $\begin{array}{l}199 \\
(5.185)\end{array}$ & $26(6.31 \%)$ & $13(8.44 \%)$ & $37(6.80 \%)$ & $\begin{array}{l}123 \\
(4.50 \%)\end{array}$ & & $\begin{array}{l}16 \\
(6.78 \%)\end{array}$ & \\
\hline Translational research & $\begin{array}{l}435 \\
(11.3 \%)\end{array}$ & $52(12.3 \%)$ & $21(13.6 \%)$ & $71(13.1 \%)$ & $\begin{array}{l}291 \\
(10.6 \%)\end{array}$ & & $\begin{array}{l}25 \\
(10.59 \%)\end{array}$ & \\
\hline Clinical duties & $\begin{array}{l}2503 \\
(65.1 \%)\end{array}$ & $241(58.5 \%)$ & 87 (56.5\%) & $324(59.7 \%)$ & $\begin{array}{l}1851 \\
(67.7 \%)\end{array}$ & & $\begin{array}{l}149 \\
(63.14 \%)\end{array}$ & \\
\hline Therapeutics/diagnostic & $69(1.80 \%)$ & $11(2.67 \%)$ & $2(1.30 \%)$ & $8(1.47 \%)$ & $\begin{array}{l}48 \\
(1.76 \%)\end{array}$ & & $4(1.69 \%)$ & \\
\hline Advocacy & $91(2.37 \%)$ & $9(2.18 \%)$ & $10(6.49 \%)$ & $20(3.68 \%)$ & $\begin{array}{l}52 \\
(1.90 \%)\end{array}$ & & 9 (3.81\%) & \\
\hline Administration & $56(1.46 \%)$ & $12(2.91 \%)$ & $5(3.25 \%)$ & $16(2.94 \%)$ & $\begin{array}{l}23 \\
(0.84 \%)\end{array}$ & & $5(2.12 \%)$ & \\
\hline Other & $51(1.33 \%)$ & $7(1.70 \%)$ & $1(0.65 \%)$ & $8(1.47 \%)$ & $\begin{array}{l}35 \\
(1.28 \%)\end{array}$ & & $2(0.85 \%)$ & \\
\hline Specialty $^{2}$ & & & & & & $<0.01$ & & 0.1632 \\
\hline Allergy and Immunology & $16(0.42 \%)$ & $2(0.49 \%)$ & $0(--)$ & $1(0.18 \%)$ & $\begin{array}{l}13 \\
(0.48 \%)\end{array}$ & & $1(0.43 \%)$ & \\
\hline Anesthesiology & $\begin{array}{l}151 \\
(3.93 \%)\end{array}$ & $18(4.43 \%)$ & $10(6.41 \%)$ & $21(3.80 \%)$ & $\begin{array}{l}102 \\
(3.75 \%)\end{array}$ & & $5(2.17 \%)$ & \\
\hline Colon and Rectal Surgery & $1(0.03 \%)$ & $0(--)$ & $1(0.03 \%)$ & $0(--)$ & $0(--)$ & & $0(--)$ & \\
\hline Dermatology & $94(2.45 \%)$ & $9(2.22 \%)$ & $4(2.56 \%)$ & $16(2.89 \%)$ & $\begin{array}{l}65 \\
(2.39 \%)\end{array}$ & & $6(2.61 \%)$ & \\
\hline Emergency Medicine & $\begin{array}{l}320 \\
(8.34 \%)\end{array}$ & $22(5.42 \%)$ & $10(6.41 \%)$ & $29(5.24 \%)$ & $\begin{array}{l}259 \\
(9.51 \%)\end{array}$ & & $\begin{array}{l}17 \\
(7.39 \%)\end{array}$ & \\
\hline Family Medicine & 285 & $22(5.42 \%)$ & $5(3.21 \%)$ & $29(5.24 \%)$ & 229 & & 21 & \\
\hline
\end{tabular}


Table 2 Career sector, career intentions, and clinical specialty interests (Continued)

\begin{tabular}{|c|c|c|c|c|c|c|c|c|}
\hline & $\begin{array}{l}\text { Total } \\
\text { n (\%) }\end{array}$ & $\begin{array}{l}\text { Asian or Pacific } \\
\text { Islander } \\
\text { n (\%) }\end{array}$ & $\begin{array}{l}\text { Black or African } \\
\text { American } \\
\text { n (\%) }\end{array}$ & $\begin{array}{l}\text { Multiracial or } \\
\text { Other } \\
\text { n (\%) }\end{array}$ & $\begin{array}{l}\text { White } \\
\text { n (\%) }\end{array}$ & $P$-value & $\begin{array}{l}\text { Hispanic } \\
\text { n (\%) }\end{array}$ & $\begin{array}{l}P \text {-value } \\
\text { (Hispanic) }\end{array}$ \\
\hline & $(7.43 \%)$ & & & & $(8.41 \%)$ & & $(9.13 \%)$ & \\
\hline Internal Medicine & $\begin{array}{l}351 \\
(9.15 \%)\end{array}$ & $44(10.8 \%)$ & $14(8.97 \%)$ & $52(9.40 \%)$ & $\begin{array}{l}241 \\
(8.85 \%)\end{array}$ & & $\begin{array}{l}15 \\
(6.52 \%)\end{array}$ & \\
\hline $\begin{array}{l}\text { Internal Medicine - } \\
\text { Cardiology }\end{array}$ & $\begin{array}{l}135 \\
(3.52 \%)\end{array}$ & 17 (4.19\%) & $9(5.77 \%)$ & $40(7.23 \%)$ & $\begin{array}{l}69 \\
(2.53 \%)\end{array}$ & & $8(3.48 \%)$ & \\
\hline $\begin{array}{l}\text { Internal Medicine - } \\
\text { Endocrinology }\end{array}$ & $21(0.83 \%)$ & $2(0.49 \%)$ & $0(--)$ & $8(1.45 \%)$ & $\begin{array}{l}22 \\
(0.81 \%)\end{array}$ & & $2(0.87 \%)$ & \\
\hline $\begin{array}{l}\text { Internal Medicine - } \\
\text { Gastroenterology }\end{array}$ & $40(1.04 \%)$ & $6(1.48 \%)$ & $2(1.28 \%)$ & $4(0.72 \%)$ & $\begin{array}{l}28 \\
(1.03 \%)\end{array}$ & & $2(0.87 \%)$ & \\
\hline $\begin{array}{l}\text { Internal Medicine } \\
\text { —-Hematology/Oncology }\end{array}$ & $\begin{array}{l}184 \\
(4.79 \%)\end{array}$ & 35 (8.62\%) & $3(1.92 \%)$ & $21(3.80 \%)$ & $\begin{array}{l}125 \\
(4.59 \%)\end{array}$ & & $8(3.48 \%)$ & \\
\hline $\begin{array}{l}\text { Internal Medicine - } \\
\text { Infectious Disease }\end{array}$ & $\begin{array}{l}103 \\
(2.68 \%)\end{array}$ & $6(1.48 \%)$ & $5(3.21 \%)$ & 10(1.81\%) & $\begin{array}{l}82 \\
(3.01 \%)\end{array}$ & & 7 (3.04\%) & \\
\hline $\begin{array}{l}\text { Internal Medicine - } \\
\text { Pulmonology }\end{array}$ & 19 (0.50\%) & $1(0.25 \%)$ & $0(--)$ & $3(0.54 \%)$ & $4(0.15 \%)$ & & $0(--)$ & \\
\hline $\begin{array}{l}\text { Internal Medicine - } \\
\text { Rheumatology }\end{array}$ & $8(0.21 \%)$ & $1(0.25 \% 0$ & $0(--)$ & $3(0.54 \% 0$ & $\begin{array}{l}4 \\
(0.15 \%)\end{array}$ & & $2(0.87 \%)$ & \\
\hline Medical Genetics & $18(0.47 \%)$ & $2(0.49 \%)$ & $0(--)$ & $1(0.18 \%)$ & $\begin{array}{l}15 \\
(0.55 \%)\end{array}$ & & $1(0.43 \%)$ & \\
\hline Neurological Surgery & $81(2.11 \%)$ & $9(2.22 \%)$ & $4(2.56 \%)$ & $15(2.71 \%)$ & $\begin{array}{l}53 \\
(1.95 \%)\end{array}$ & & $6(2.61 \%)$ & \\
\hline Neurology & $\begin{array}{l}170 \\
(4.43 \%)\end{array}$ & $18(4.43 \%)$ & $2(1.28 \%)$ & $27(4.88 \%)$ & $\begin{array}{l}123 \\
(4.52 \%)\end{array}$ & & $\begin{array}{l}11 \\
(4.78 \%)\end{array}$ & \\
\hline Nuclear Medicine & $3(0.08 \%)$ & $0(--)$ & $0(--)$ & $0(--)$ & $\begin{array}{l}3 \\
(0.11 \%)\end{array}$ & & $0(--)$ & \\
\hline Obstetrics and Gynecology & $\begin{array}{l}180 \\
(4.69 \%)\end{array}$ & $14(3.45 \%)$ & $11(7.05 \%)$ & 25 (4.52\%) & $\begin{array}{l}130 \\
(4.77 \%)\end{array}$ & & $\begin{array}{l}16 \\
(6.96 \%)\end{array}$ & \\
\hline Ophthalmology & $\begin{array}{l}114 \\
(2.97 \%)\end{array}$ & $21(5.17 \%)$ & $0(--)$ & $28(5.06 \%)$ & $\begin{array}{l}65 \\
(2.39 \%)\end{array}$ & & $5(2.17 \%)$ & \\
\hline Orthopedic Surgery & $\begin{array}{l}147 \\
(3.83 \%)\end{array}$ & $14(3.45 \%)$ & $5(3.21 \%)$ & $25(4.52 \%)$ & $\begin{array}{l}103 \\
(3.78 \%)\end{array}$ & & $\begin{array}{l}15 \\
(6.52 \%)\end{array}$ & \\
\hline Otolaryngology & 79 (2.06\%) & $7(1.72 \%)$ & $1(0.64 \%)$ & $18(3.25 \%)$ & $\begin{array}{l}53 \\
(1.95 \%)\end{array}$ & & $3(1.30 \%)$ & \\
\hline Pathology & $56(1.46 \%)$ & $6(1.48 \%)$ & $3(1.92 \%)$ & $6(1.08 \%)$ & $\begin{array}{l}41 \\
(1.51 \%)\end{array}$ & & $1(0.43 \%)$ & \\
\hline Pediatrics & $\begin{array}{l}495 \\
(12.90 \%)\end{array}$ & $47(11.58 \%)$ & $26(16.7 \%)$ & $58(10.5 \%)$ & $\begin{array}{l}364 \\
(13.4 \%)\end{array}$ & & $\begin{array}{l}27 \\
(11.74 \%)\end{array}$ & \\
\hline $\begin{array}{l}\text { Physical Medicine and } \\
\text { Rehabilitation }\end{array}$ & 35 (0.91\%) & $2(0.49 \%)$ & $2(1.28 \%)$ & $7(.1 .27 \%)$ & $\begin{array}{l}24 \\
(0.88 \%)\end{array}$ & & $3(1.30 \%)$ & \\
\hline Plastic Surgery & 40 (1.04\%) & $5(1.23 \%)$ & $1(0.64 \%)$ & $2(0.36 \%)$ & $\begin{array}{l}32 \\
(1.18 \%)\end{array}$ & & $2(0.87 \%)$ & \\
\hline Preventative Medicine & $12(0.31 \%)$ & $5(1.23 \%)$ & $1(0.64 \%)$ & $3(0.54 \%)$ & $\begin{array}{l}3 \\
(0.11 \%)\end{array}$ & & $1(0.43 \%)$ & \\
\hline Psychiatry & $\begin{array}{l}114 \\
(2.97 \%)\end{array}$ & $7(1.72 \%)$ & $8(5.13 \%)$ & $14(2.53 \%)$ & $\begin{array}{l}85 \\
(3.12 \%)\end{array}$ & & $4(1.74 \%)$ & \\
\hline Radiation Oncology & $54(1.41 \%)$ & $13(3.20 \%)$ & $5(3.21 \%)$ & $6(1.08 \%)$ & $\begin{array}{l}30 \\
(1.10 \%)\end{array}$ & & $1(0.43 \%)$ & \\
\hline Radiology & $\begin{array}{l}107 \\
(2.79 \%)\end{array}$ & $9(2.22 \%)$ & $4(2.56 \%)$ & 22 (3.98\%) & $\begin{array}{l}72 \\
(2.64 \%)\end{array}$ & & $2(0.87 \%)$ & \\
\hline Surgery & $237(6.18 \%)$ & $23(5.67 \%)$ & $12(7.69 \%)$ & 34 (6.15\%) & $\begin{array}{l}168 \\
(6.17 \%)\end{array}$ & & $\begin{array}{l}21 \\
(9.13 \%)\end{array}$ & \\
\hline Thoracic Surgery & $31(0.81 \%)$ & $1(0.25 \% 0$ & $1(0.64 \%)$ & $8(1.45 \%)$ & 21 & & $3(1.30 \%)$ & \\
\hline
\end{tabular}


Table 2 Career sector, career intentions, and clinical specialty interests (Continued)

\begin{tabular}{lllllll}
\hline & $\begin{array}{l}\text { Total } \\
\mathbf{n}(\%)\end{array}$ & $\begin{array}{l}\text { Asian or Pacific } \\
\text { Islander } \\
\mathbf{n}(\%)\end{array}$ & $\begin{array}{l}\text { Black or African } \\
\text { American } \\
\mathbf{n}(\%)\end{array}$ & $\begin{array}{l}\text { Multiracial or } \\
\text { Other } \\
\mathbf{n}(\%)\end{array}$ & $\begin{array}{l}\text { White } \\
\mathbf{n}(\%)\end{array}$ & $\begin{array}{l}P \text {-value } \\
\text { Hispanic P-value } \\
\mathbf{n}(\%)\end{array}$ \\
Urologispanic)
\end{tabular}

\footnotetext{
${ }^{\text {a }}$ Fisher's Exact calculated due to minimum cell count violations

${ }^{1}$ Respondents could select all applicable choices, will not sum to $100 \%$

${ }^{2}$ Respondents could select up to TWO choices, will not sum to $100 \%$

${ }^{3}$ Respondents could select up to THREE choices, will not sum to $100 \%$
}

intention between Hispanics and non-Hispanics. There were also no significant differences between Asians and Whites across all career intention comparisons.

\section{Experienced and anticipated obstacles}

Trainees of all ethnic backgrounds reported balancing family and work responsibilities as the top obstacle experienced during training, with balancing clinic, research, and education responsibilities as the second biggest challenge (Table 4). Black/African American trainees were most likely to describe experiencing discrimination and bias (18.6\%). Rates of discrimination and bias as an experienced obstacle among Black/African American trainees ranged from 1.9 to 3.4-fold greater than trainees from other racial/ethnic groups.

All trainees reported balancing family and work responsibilities as the top predicted obstacle (Table 4). Following this, Asian (12.5\%) and Multiracial/Other (12.3\%) indicated not finding a position in a desired location, whereas Black/African American (17.4\%), Hispanic (16.1\%), and White (15.2\%) trainees selected loan repayment as the next most predicted obstacle. As with experienced obstacles, Black/African American trainees were also most likely to select discrimination and bias as a predicted obstacle, with a rate ranging from 1.5 to 7.2fold greater than those of trainees of other races/ ethnicities.

Whereas perceptions of research feasibility in surgical specialties did not significantly differ across racial/ethnic groups, perceptions of research feasibility in acute care medicine specialties were different (Table 5). In particular, $62.3 \%$ of White, $61.1 \%$ of Black/African American, $60.6 \%$ of Multiracial/Other, $60.1 \%$ of Hispanic, and $59 \%$ of Asian trainees viewed research-intensive careers in acute care medical specialties as difficult, highly difficult, or impossible. Conversely, Black/African American (7.6\%) and Multiracial/Other (7.4\%) trainees were most likely to view research intense careers in acute care medical specialties as highly feasible. In terms of feasibility of research intense careers in surgical specialties, there was a non-statistically significant trend toward fewer Black/ African American trainees rating research intense careers in surgery to be difficult, highly difficult, or impossible (60.6\%) as compared to Asian (64.8\%), Multiracial/

Table 3 Logistic regression on career plan after residency, adjusting for training stage, sex, career sector intentions, specialty intentions, and mentorship status

\begin{tabular}{|c|c|c|c|c|c|c|c|}
\hline \multirow[b]{2}{*}{ Career Plan } & \multicolumn{7}{|c|}{ Effects of ethnicity on career intention, OR (95\% CI) } \\
\hline & $\begin{array}{l}\text { Asian or Pacific } \\
\text { Islander vs. Black or } \\
\text { African American } \\
n=590\end{array}$ & $\begin{array}{l}\text { Asian or Pacific } \\
\text { Islander vs. Black of } \\
\text { Multiracial or Other } \\
n=1166\end{array}$ & $\begin{array}{l}\text { Asian or Pacific } \\
\text { Islander vs. } \\
\text { White } n=3276\end{array}$ & $\begin{array}{l}\text { Black or African } \\
\text { American vs. } \\
\text { Multiracial or } \\
\text { Other } \\
n=742\end{array}$ & $\begin{array}{l}\text { Black or } \\
\text { African } \\
\text { American vs. } \\
\text { White } \\
n=3018\end{array}$ & $\begin{array}{l}\text { Multiracial } \\
\text { or Other } \\
\text { vs. White } \\
n=3428\end{array}$ & $\begin{array}{l}\text { Hispanic } \\
\text { vs. Not } \\
\text { Hispanic } \\
n=2945\end{array}$ \\
\hline Academia & $1.693(1.031-2.781)$ & $1.100(0.775-1.563)$ & $\begin{array}{l}0.840(0.628- \\
1.123)\end{array}$ & $0.650(0.404-1.045)$ & $\begin{array}{l}0.496(0.322- \\
0.764)\end{array}$ & $\begin{array}{l}0.763 \\
(0.594- \\
0.980)\end{array}$ & $\begin{array}{l}1.087 \\
(0.741- \\
1.596)\end{array}$ \\
\hline $\begin{array}{l}\text { Basic } \\
\text { Research }\end{array}$ & $3.229(1.123-9.281)$ & $1.475(0.873-2.492)$ & $\begin{array}{l}1.013(0.673- \\
1.527)\end{array}$ & $0.457(0.158-1.317)$ & $\begin{array}{l}0.314(0.115- \\
0.857)\end{array}$ & $\begin{array}{l}0.687 \\
(0.457- \\
1.034)\end{array}$ & $\begin{array}{l}1.289 \\
(0.688- \\
2.414)\end{array}$ \\
\hline $\begin{array}{l}\text { Translational } \\
\text { Research }\end{array}$ & $1.113(0.692-1.790)$ & $0.922(0.518-1.642)$ & $\begin{array}{l}1.372(0.909- \\
2.070)\end{array}$ & $\begin{array}{l}1.546(1.107- \\
2.159)\end{array}$ & $\begin{array}{l}1.488(0.844- \\
2.624)\end{array}$ & $\begin{array}{l}1.677 \\
(1.004- \\
2.799)\end{array}$ & $\begin{array}{l}1.126 \\
(0.825- \\
1.539)\end{array}$ \\
\hline $\begin{array}{l}\text { Clinical } \\
\text { Research }\end{array}$ & $1.292(0.770-2.165)$ & $1.057(0.750-1.489)$ & $\begin{array}{l}0.991(0.749- \\
1.312)\end{array}$ & $0.818(0.495-1.352)$ & $\begin{array}{l}0.768(0.485- \\
1.215)\end{array}$ & $\begin{array}{l}0.938 \\
(0.731- \\
1.203)\end{array}$ & $\begin{array}{l}1.207 \\
(0.704- \\
1.499)\end{array}$ \\
\hline
\end{tabular}


Table 4 Career and non-career related responsibilities, obstacles, and factors in career selection

\begin{tabular}{|c|c|c|c|c|c|c|c|c|}
\hline & $\begin{array}{l}\text { Total } \\
\text { n (\%) }\end{array}$ & $\begin{array}{l}\text { Asian or Pacific } \\
\text { Islander } \\
\text { n (\%) }\end{array}$ & $\begin{array}{l}\text { Black or African } \\
\text { American } \\
\mathrm{n}(\%)\end{array}$ & $\begin{array}{l}\text { Multiracial or } \\
\text { Other } \\
\mathrm{n}(\%)\end{array}$ & $\begin{array}{l}\text { White } \\
\text { n (\%) }\end{array}$ & $\begin{array}{l}P \text { - } \\
\text { value }\end{array}$ & $\begin{array}{l}\text { Hispanic } \\
\text {, n (\%) }\end{array}$ & $\begin{array}{l}P \text {-value } \\
\text { (Hispanic) }\end{array}$ \\
\hline \multicolumn{9}{|c|}{ Foreseeable non-work-related responsibilities DURING residency $\mathbf{n}$ (\% of $\mathbf{4 4 3 3}$ Total) } \\
\hline Raising children & $\begin{array}{l}2573 \\
(63.8 \%)\end{array}$ & $256(60.0 \%)$ & $104(62.3 \%)$ & $331(57.4 \%)$ & $\begin{array}{l}1882 \\
(65.8 \%)\end{array}$ & .0004 & $\begin{array}{l}142 \\
(58.7 \%)\end{array}$ & 0.0990 \\
\hline Taking care of elderly parents & $\begin{array}{l}760 \\
(18.8 \%)\end{array}$ & $130(30.4 \%)$ & $32(19.2 \%)$ & $166(28.8 \%)$ & $\begin{array}{l}432 \\
(15.1 \%)\end{array}$ & $<.0001$ & $\begin{array}{l}52 \\
(21.5 \%)\end{array}$ & 0.3374 \\
\hline Being caretaker to others & $\begin{array}{l}562 \\
(13.9 \%)\end{array}$ & $79(18.5 \%)$ & $31(18.6 \%)$ & $108(18.7 \%)$ & $\begin{array}{l}344 \\
(12.0 \%)\end{array}$ & $<.0001$ & $\begin{array}{l}49 \\
(20.3 \%)\end{array}$ & 0.0048 \\
\hline Financial support of others & $\begin{array}{l}1095 \\
(27.2 \%)\end{array}$ & $127(29.7 \%)$ & 75 (44.9\%) & $193(33.5 \%)$ & $\begin{array}{l}700 \\
(24.5 \%)\end{array}$ & $<.0001$ & $\begin{array}{l}84 \\
(34.7 \%)\end{array}$ & 0.0093 \\
\hline \multicolumn{9}{|c|}{ Foreseeable non-work-related responsibilities AFTER residency ${ }^{1}$} \\
\hline Raising children & $\begin{array}{l}3572 \\
(88.6 \%)\end{array}$ & $373(87.4 \%)$ & $141(84.4 \%)$ & $508(88.0 \%)$ & $\begin{array}{l}2550 \\
(89.1 \%)\end{array}$ & .2215 & $\begin{array}{l}209 \\
(86.4 \%)\end{array}$ & 0.2949 \\
\hline Taking care of elderly parents & $\begin{array}{l}2534 \\
(65.0 \%)\end{array}$ & $326(76.35 \%)$ & $100(59.9 \%)$ & 409 (70.9\%) & $\begin{array}{l}1788 \\
(62.5 \%)\end{array}$ & $<.0001$ & $\begin{array}{l}143 \\
(59.1 \%)\end{array}$ & 0.0446 \\
\hline Being caretaker to others & $\begin{array}{l}1224 \\
(30.4 \%)\end{array}$ & $168(39.3 \%)$ & $51(30.5 \%)$ & $215(37.3 \%)$ & $\begin{array}{l}790 \\
(27.6 \%)\end{array}$ & $<.0001$ & $\begin{array}{l}75 \\
(31.0 \%)\end{array}$ & 0.8877 \\
\hline Financial support of others & $\begin{array}{l}2152 \\
(53.4 \%)\end{array}$ & $241(56.4 \%)$ & $101(60.5 \%)$ & $338(58.6 \%)$ & $\begin{array}{l}1472 \\
(51.4 \%)\end{array}$ & 0.0014 & $\begin{array}{l}155 \\
(64.1 \%)\end{array}$ & 0.0008 \\
\hline \multicolumn{9}{|l|}{ Experienced Obstacles ${ }^{1}$} \\
\hline $\begin{array}{l}\text { Balancing family and work } \\
\text { responsibilities }\end{array}$ & $\begin{array}{l}1548 \\
(38.4 \%)\end{array}$ & $154(36.1 \%)$ & $55(32.9 \%)$ & 195 (33.8\%) & $\begin{array}{l}1144 \\
(39.97 \%)\end{array}$ & 0.0104 & $\begin{array}{l}109 \\
(45.04 \%)\end{array}$ & 0.0283 \\
\hline $\begin{array}{l}\text { Balance clinical, research, \& } \\
\text { education responsibilities }\end{array}$ & $\begin{array}{l}879 \\
(21.8 \%)\end{array}$ & $106(24.8 \%)$ & $36(21.6 \%)$ & $129(22.4 \%)$ & $\begin{array}{l}608 \\
(21.2 \%)\end{array}$ & .4039 & $\begin{array}{l}60 \\
(24.79 \%)\end{array}$ & 0.2425 \\
\hline Loan repayment & $\begin{array}{l}756 \\
(18.8 \%)\end{array}$ & $78(18.2 \%)$ & $23(13.8 \%)$ & $90(15.6 \%)$ & $\begin{array}{l}565 \\
(19.7 \%)\end{array}$ & 0.0386 & $\begin{array}{l}60 \\
(24.8 \%)\end{array}$ & 0.0138 \\
\hline Lack of opportunity/funding & $\begin{array}{l}699 \\
(17.3 \%)\end{array}$ & $81(19.0 \%)$ & $28(16.8 \%)$ & $105(18.2 \%)$ & $\begin{array}{l}485 \\
(17.0 \%)\end{array}$ & .6972 & $\begin{array}{l}56 \\
(23.1 \%)\end{array}$ & 0.0164 \\
\hline $\begin{array}{l}\text { Satisfactory professional } \\
\text { development }\end{array}$ & $\begin{array}{l}375 \\
(9.30 \%)\end{array}$ & $50(11.7 \%)$ & $17(10.2 \%)$ & $70(12.1 \%)$ & $\begin{array}{l}283 \\
(8.32 \%)\end{array}$ & .0079 & $\begin{array}{l}28 \\
(11.57 \%)\end{array}$ & 0.2411 \\
\hline Under-compensation & $\begin{array}{l}263 \\
(6.52 \%)\end{array}$ & 27 (6.32\%) & $5(2.99 \%)$ & $34(5.89 \%)$ & $\begin{array}{l}197 \\
(6.88 \%)\end{array}$ & .2190 & $\begin{array}{l}18 \\
(7.44 \%)\end{array}$ & 0.5717 \\
\hline $\begin{array}{l}\text { Discrimination/biases (gender/ } \\
\text { ethnicity) }\end{array}$ & $\begin{array}{l}276 \\
(6.84 \%)\end{array}$ & $41(9.60 \%)$ & $31(18.6 \%)$ & $46(7.97 \%)$ & $\begin{array}{l}158 \\
(5.52 \%)\end{array}$ & $<.0001$ & $\begin{array}{l}22 \\
(9.09 \%)\end{array}$ & 0.1779 \\
\hline $\begin{array}{l}\text { Not finding position in desired } \\
\text { location }\end{array}$ & $\begin{array}{l}318 \\
(7.88 \%)\end{array}$ & 49 (11.5\%) & $8(4.79 \%)$ & 60 (10.4\%) & $\begin{array}{l}201 \\
(7.02 \%)\end{array}$ & 0.0005 & $\begin{array}{l}11 \\
(4.55 \%)\end{array}$ & 0.0401 \\
\hline Sexual harassment & $\begin{array}{l}49 \\
(1.21 \%)\end{array}$ & $4(0.94 \%)$ & $2(1.20 \%)$ & $9(1.56 \%)$ & $\begin{array}{l}34 \\
(1.19 \%)\end{array}$ & .8339 & $8(3.31 \%)$ & 0.0028 \\
\hline Malpractice/lawsuit & $\begin{array}{l}26 \\
(0.64 \%)\end{array}$ & $1(0.23 \%)$ & $1(0.60 \%)$ & $4(0.69 \%)$ & $\begin{array}{l}20 \\
(0.70 \%)\end{array}$ & .7337 & $1(0.41 \%)$ & 0.6018 \\
\hline $\begin{array}{l}\text { Predicted Obstacles }{ }^{1} \mathbf{n} \text { (\% of } \mathbf{3 8 1 9} \\
\text { Total) }\end{array}$ & & & & & & $<.0001$ & & 0.0634 \\
\hline $\begin{array}{l}\text { Balancing family and work } \\
\text { responsibilities }\end{array}$ & $\begin{array}{l}1907 \\
(49.9 \%)\end{array}$ & $194(47.6 \%)$ & $60(38.7 \%)$ & $252(46.4 \%)$ & $\begin{array}{l}1401 \\
(51.6 \%)\end{array}$ & & $\begin{array}{l}112 \\
(47.46 \%)\end{array}$ & \\
\hline $\begin{array}{l}\text { Balance clinical, research, \& } \\
\text { education responsibilities }\end{array}$ & $\begin{array}{l}225 \\
(8.29 \%)\end{array}$ & $36(8.82 \%)$ & $18(11.6 \%)$ & $59(10.9 \%)$ & $\begin{array}{l}225 \\
(8.29 \%)\end{array}$ & & $\begin{array}{l}21 \\
(8.90 \%)\end{array}$ & \\
\hline $\begin{array}{l}\text { Not finding position in desired } \\
\text { location }\end{array}$ & $\begin{array}{l}358 \\
(9.37 \%)\end{array}$ & $51(12.5 \%)$ & $20(12.90 \%)$ & 67 (12.34\%) & $\begin{array}{l}220 \\
(8.11 \%)\end{array}$ & & $\begin{array}{l}16 \\
(6.78 \%)\end{array}$ & \\
\hline Loan repayment & $\begin{array}{l}517 \\
(13.5 \%)\end{array}$ & $26(6.37 \%)$ & $27(17.4 \%)$ & $51(9.39 \%)$ & $\begin{array}{l}413 \\
(15.2 \%)\end{array}$ & & $\begin{array}{l}38 \\
(16.10 \%)\end{array}$ & \\
\hline Under-compensation & $\begin{array}{l}137 \\
(3.59 \%)\end{array}$ & $12(2.94 \%)$ & $5(3.23 \%)$ & $20(2.8 \%)$ & $\begin{array}{l}100 \\
(3.69 \%)\end{array}$ & & $\begin{array}{l}13 \\
(5.51 \%)\end{array}$ & \\
\hline
\end{tabular}


Table 4 Career and non-career related responsibilities, obstacles, and factors in career selection (Continued)

\begin{tabular}{|c|c|c|c|c|c|c|c|c|}
\hline & $\begin{array}{l}\text { Total } \\
\text { n (\%) }\end{array}$ & $\begin{array}{l}\text { Asian or Pacific } \\
\text { Islander } \\
\text { n (\%) }\end{array}$ & $\begin{array}{l}\text { Black or African } \\
\text { American } \\
\mathrm{n}(\%)\end{array}$ & $\begin{array}{l}\text { Multiracial or } \\
\text { Other } \\
\text { n (\%) }\end{array}$ & $\begin{array}{l}\text { White } \\
\text { n (\%) }\end{array}$ & $\begin{array}{l}P \text { - } \\
\text { value }\end{array}$ & $\begin{array}{l}\text { Hispanic } \\
\text {, n (\%) }\end{array}$ & $\begin{array}{l}P \text {-value } \\
\text { (Hispanic) }\end{array}$ \\
\hline Lack of opportunity/funding & $\begin{array}{l}322 \\
(8.43 \%)\end{array}$ & $45(11.0 \%)$ & $7(4.52 \%)$ & 54 (9.94\%) & $\begin{array}{l}216 \\
(7.96 \%)\end{array}$ & & $\begin{array}{l}17 \\
(7.20 \%)\end{array}$ & \\
\hline Malpractice/lawsuit & $\begin{array}{l}55 \\
(1.44 \%)\end{array}$ & $6(1.47 \%)$ & $4(2.58 \%)$ & $6(1.10 \%)$ & $\begin{array}{l}39 \\
(1.44 \%)\end{array}$ & & $3(1.27 \%)$ & \\
\hline $\begin{array}{l}\text { Satisfactory professional } \\
\text { development }\end{array}$ & $\begin{array}{l}98 \\
(2.57 \%)\end{array}$ & $19(4.66 \%)$ & $5(3.23 \%)$ & $18(3.31 \%)$ & $\begin{array}{l}56 \\
(2.06 \%)\end{array}$ & & $7(2.97 \%)$ & \\
\hline $\begin{array}{l}\text { Discrimination/biases (gender/ } \\
\text { ethnicity) }\end{array}$ & $\begin{array}{l}44 \\
(1.15 \%)\end{array}$ & $9(2.21 \%)$ & $7(4.52 \%)$ & $11(2.03 \%)$ & $\begin{array}{l}17 \\
(0.63 \%)\end{array}$ & & $7(2.97 \%)$ & \\
\hline Sexual harassment & $\begin{array}{l}3 \\
(0.08 \%)\end{array}$ & $1(0.25 \%)$ & $1(0.65 \%)$ & $0(--)$ & $1(0.04 \%)$ & & $1(0.42 \%)$ & \\
\hline Other & $\begin{array}{l}40 \\
(1.05 \%)\end{array}$ & $9(2.21 \%)$ & $1(0.65 \%)$ & $5(0.92 \%)$ & $\begin{array}{l}25 \\
(0.92 \%)\end{array}$ & & $1(0.42 \%)$ & \\
\hline $\begin{array}{l}\text { Most important factors in career } \\
\text { selection } \\
\mathbf{n} \text { (\% of } \mathbf{3 8 3 3} \text { Total) }\end{array}$ & & & & & & 0.0003 & & \\
\hline $\begin{array}{l}\text { Ability to balance work \& personal } \\
\text { life }\end{array}$ & $\begin{array}{l}1371 \\
(35.7 \%)\end{array}$ & $151(36.7 \%)$ & $52(33.6 \%)$ & $180(32.9 \%)$ & $\begin{array}{l}988 \\
(36.3 \%)\end{array}$ & & $\begin{array}{l}92 \\
(39.32 \%)\end{array}$ & \\
\hline Opportunities for patient care & $\begin{array}{l}1303 \\
(34.0 \%)\end{array}$ & $132(32.0 \%)$ & $46(29.7 \%)$ & $164(30.0 \%)$ & $\begin{array}{l}961 \\
(35.3 \%)\end{array}$ & & $\begin{array}{l}71 \\
(30.34 \%)\end{array}$ & \\
\hline Financial security & $\begin{array}{l}160 \\
(4.17 \%)\end{array}$ & $18(4.73 \%)$ & $9(5.81 \%)$ & $35(6.40 \%)$ & $\begin{array}{l}98 \\
(3.60 \%)\end{array}$ & & $\begin{array}{l}16 \\
(6.84 \%)\end{array}$ & \\
\hline Opportunities to teach & $\begin{array}{l}101 \\
(2.63 \%)\end{array}$ & $12(2.91 \%)$ & $3(1.94 \%)$ & $15(2.74 \%)$ & $\begin{array}{l}71 \\
(2.61 \%)\end{array}$ & & $2(0.85 \%$ & 0.4831 \\
\hline Opportunities for research & $\begin{array}{l}414 \\
(10.8 \%)\end{array}$ & $48(11.7 \%)$ & $12(7.74 \%)$ & $61(11.2 \%)$ & $\begin{array}{l}293 \\
(10.8 \%)\end{array}$ & & $\begin{array}{l}24 \\
(10.26 \%)\end{array}$ & \\
\hline $\begin{array}{l}\text { Opportunities for community } \\
\text { service }\end{array}$ & $\begin{array}{l}122 \\
(3.18 \%)\end{array}$ & $10(2.43 \%)$ & $13(1.94 \%)$ & $17(3.11 \%)$ & $\begin{array}{l}82 \\
(3.01 \%)\end{array}$ & & $9(3.85 \%)$ & \\
\hline $\begin{array}{l}\text { Opportunities for international } \\
\text { work }\end{array}$ & $\begin{array}{l}109 \\
(2.84 \%)\end{array}$ & $11(2.67 \%)$ & $11(7.10 \%)$ & $21(3.84 \%)$ & $\begin{array}{l}66 \\
(2.42 \%)\end{array}$ & & $1(0.43 \%)$ & \\
\hline Autonomy & $\begin{array}{l}89 \\
(2.32 \%)\end{array}$ & $14(3.40 \%)$ & $2(1.29 \%)$ & $20(3.66 \%)$ & $\begin{array}{l}53 \\
(1.95 \%)\end{array}$ & & $5(2.14 \%)$ & \\
\hline $\begin{array}{l}\text { Opportunities for student } \\
\text { interactions }\end{array}$ & $\begin{array}{l}36 \\
(0.94 \%)\end{array}$ & $1(0.24 \%)$ & $2(1.29 \%)$ & $6(1.10 \%)$ & $\begin{array}{l}27 \\
(0.99 \%)\end{array}$ & & $3(1.28 \%)$ & \\
\hline Prestige & $\begin{array}{l}14 \\
(0.36 \%)\end{array}$ & $1(0.24 \%)$ & $0(--)$ & $4(0.73 \%)$ & $9(0.33 \%)$ & & $1(0.43 \%)$ & \\
\hline Opportunities for travel & $\begin{array}{l}24 \\
(0.63 \%)\end{array}$ & $3(0.73 \%)$ & $1(0.65 \%)$ & $4(0.73 \%)$ & $\begin{array}{l}16 \\
(0.59 \%)\end{array}$ & & $1(0.43 \%)$ & \\
\hline Opportunities for local work & $\begin{array}{l}19 \\
(0.50 \%)\end{array}$ & $1(0.24 \%)$ & $0(--)$ & $7(1.28 \%)$ & $\begin{array}{l}11 \\
(0.40 \%)\end{array}$ & & $1(0.43 \%)$ & \\
\hline Opportunities for national work & $\begin{array}{l}16 \\
(0.42 \%)\end{array}$ & $4(0.97 \%)$ & $2(1.29 \%)$ & $2(0.37 \%)$ & $8(0.29 \%)$ & & $1(0.43 \%)$ & \\
\hline
\end{tabular}

${ }^{a}$ Fisher's Exact calculated due to minimum cell count violations

${ }^{1}$ Respondents could select all applicable choices, will not sum to $100 \%$

${ }^{2}$ Respondents could select up to TWO choices, will not sum to $100 \%$

${ }^{3}$ Respondents could select up to THREE choices, will not sum to $100 \%$

Other (62.8\%), White (63.1\%) and Hispanic (65.1\%) trainees.

\section{Importance of mentoring and professional networking}

The majority of trainees in each racial/ethnic group were able to identify a mentor who helped them progress toward and/or achieve their career goals (Table 5).
However, perceptions in the value of mentorship varied between groups, with Black/African American trainees (97.6\%) most likely to view mentorship as very or somewhat important in their training compared to other trainee groups (Asian 86.2\%; Multiracial/Other 91.5\%; White 87.9\%; Hispanic 88.7\%). Conversely, Asian trainees $(13.8 \%)$ were most likely to describe mentors as 
Table 5 Perceptions of research feasibility and the importance of mentoring

\begin{tabular}{|c|c|c|c|c|c|c|c|c|}
\hline & $\begin{array}{l}\text { Total, } \\
\text { n (\%) }\end{array}$ & $\begin{array}{l}\text { Asian or } \\
\text { Pacific } \\
\text { Islander, } \mathrm{n} \\
(\%)\end{array}$ & $\begin{array}{l}\text { Black or } \\
\text { African } \\
\text { American, } \\
\mathrm{n}(\%)\end{array}$ & $\begin{array}{l}\text { Multiracial } \\
\text { or Other, } \mathrm{n} \\
\text { (\%) }\end{array}$ & $\begin{array}{l}\text { White, } \\
\text { n (\%) }\end{array}$ & $\begin{array}{l}P- \\
\text { value }\end{array}$ & $\begin{array}{l}\text { Hispanic, } \\
\text { n (\%) }\end{array}$ & $\begin{array}{l}\text { Hispanic } \\
P \text {-value }\end{array}$ \\
\hline $\begin{array}{l}\text { How feasible is a research intense career in acute care } \\
\text { medicine specialties? }\end{array}$ & & & & & & 0.0315 & & 0.4490 \\
\hline Highly feasible & $\begin{array}{l}244 \\
(6.33 \%)\end{array}$ & $18(4.41 \%)$ & $12(7.64 \%)$ & $41(7.43 \%)$ & $\begin{array}{l}173 \\
(6.32 \%)\end{array}$ & & $15(6.44 \%)$ & \\
\hline Feasible & $\begin{array}{l}1234 \\
(32.0 \%)\end{array}$ & 149 (36.5\%) & 49 (31.2\%) & $177(32.1 \%)$ & $\begin{array}{l}859 \\
(31.4 \%)\end{array}$ & & $\begin{array}{l}78 \\
(33.48 \%)\end{array}$ & \\
\hline Difficult & $\begin{array}{l}1605 \\
(41.6 \%)\end{array}$ & $176(43.1 \%)$ & $74(47.1 \%)$ & 209 (37.9\%) & $\begin{array}{l}1146 \\
(41.8 \%)\end{array}$ & & $\begin{array}{l}103 \\
(44.21 \%)\end{array}$ & \\
\hline Highly difficult & $\begin{array}{l}711 \\
(18.4 \%)\end{array}$ & $59(14.5 \%)$ & $22(14.0 \%)$ & $119(21.6 \%)$ & $\begin{array}{l}511 \\
(18.7 \%)\end{array}$ & & $\begin{array}{l}32 \\
(13.73 \%\end{array}$ & \\
\hline Impossible & $\begin{array}{l}62 \\
(1.61 \%)\end{array}$ & $6(1.47 \%)$ & $0(--)$ & $6(1.09 \%)$ & $\begin{array}{l}50 \\
(1.83 \%)\end{array}$ & & $5(2.15 \%)$ & \\
\hline TOTAL & $\begin{array}{l}3856 \\
(100 \%)\end{array}$ & $408(100 \%)$ & 157 (100\%) & $552(100 \%)$ & $\begin{array}{l}2739 \\
(100 \%)\end{array}$ & & $\begin{array}{l}233 \\
(100 \%)\end{array}$ & \\
\hline $\begin{array}{l}\text { How feasible is a research intense career in surgical } \\
\text { specialties? }\end{array}$ & & & & & & 0.0538 & & 0.7247 \\
\hline Highly feasible & $\begin{array}{l}253 \\
(6.56 \%)\end{array}$ & $30(7.35 \%)$ & $7(4.46 \%)$ & $48(8.71 \%)$ & $\begin{array}{l}168 \\
(6.13 \%)\end{array}$ & & 17 (7.33\%) & \\
\hline Feasible & $\begin{array}{l}1169 \\
(30.3 \%)\end{array}$ & $114(27.9 \%)$ & $55(35.0 \%)$ & $157(28.5 \%)$ & $\begin{array}{l}843 \\
(30.8 \%)\end{array}$ & & $\begin{array}{l}64 \\
(27.59 \%)\end{array}$ & \\
\hline Difficult & $\begin{array}{l}1350 \\
(35.0 \%)\end{array}$ & $140(34.3 \%)$ & 61 (38.9\%) & $168(30.5 \%)$ & $\begin{array}{l}981 \\
(35.8 \%)\end{array}$ & & $\begin{array}{l}79 \\
(34.05 \%)\end{array}$ & \\
\hline Highly difficult & $\begin{array}{l}947 \\
(24.6 \%)\end{array}$ & 107 (26.3\%) & $29(18.5 \%)$ & $157(28.5 \%)$ & $\begin{array}{l}654 \\
(23.9 \%)\end{array}$ & & ${ }^{61}(26.29 \%$ & \\
\hline Impossible & $\begin{array}{l}137 \\
(3.55 \%)\end{array}$ & $17(4.17 \%)$ & $5(3.18 \%)$ & $21(3.81 \%)$ & $\begin{array}{l}94 \\
(3.43 \%)\end{array}$ & & $11(4.74 \%)$ & \\
\hline TOTAL & $\begin{array}{l}3856 \\
(100 \%)\end{array}$ & $408(100 \%)$ & 157 (100\%) & $551(100 \%)$ & $\begin{array}{l}2704 \\
(100 \%)\end{array}$ & & $\begin{array}{l}232 \\
(100 \%\end{array}$ & \\
\hline $\begin{array}{l}\text { Can you currently identify a mentor(s) who has helped } \\
\text { you progress toward } \& \text { /or achieve your career goals? }\end{array}$ & & & & & & 0.1445 & & 0.6488 \\
\hline Yes & $\begin{array}{l}2890 \\
(76.3 \%)\end{array}$ & 322 (78.9\%) & 125 (82.2\%) & 409 (76.5\%) & $\begin{array}{l}2034 \\
(75.5 \%)\end{array}$ & & $\begin{array}{l}181 \\
(77.7 \%)\end{array}$ & \\
\hline No & $\begin{array}{l}898 \\
(23.7 \%)\end{array}$ & $86(21.1 \%)$ & $27(17.8 \%)$ & $126(23.6 \%)$ & $\begin{array}{l}659 \\
(24.5 \%)\end{array}$ & & $52(22.3 \%)$ & \\
\hline TOTAL & $\begin{array}{l}3788 \\
(100 \%)\end{array}$ & $408(100 \%)$ & $152(100 \%)$ & $535(100 \%)$ & $\begin{array}{l}2693 \\
(100 \%)\end{array}$ & & $\begin{array}{l}233 \\
(100 \%)\end{array}$ & \\
\hline $\begin{array}{l}\text { How important has mentorship been in your training } \\
\text { thus far? }\end{array}$ & & & & & & $<.0001$ & & 0.3868 \\
\hline Very important & $\begin{array}{l}1274 \\
(44.5 \%)\end{array}$ & $145(45.6 \%)$ & $72(57.6 \%)$ & $214(52.5 \%)$ & $\begin{array}{l}843 \\
(41.8 \%)\end{array}$ & & $89(50.3 \%)$ & \\
\hline Somewhat important & $\begin{array}{l}1267 \\
(44.2 \%)\end{array}$ & $129(40.6 \%)$ & $50(40.0 \%)$ & $159(39.0 \%)$ & $\begin{array}{l}929 \\
(46.1 \%)\end{array}$ & & $68(38.4 \%)$ & \\
\hline Not very important & $\begin{array}{l}300 \\
(10.5 \%)\end{array}$ & $42(13.2 \%)$ & $3(2.40 \%)$ & $32(7.48 \%)$ & $\begin{array}{l}223 \\
(11.1 \%)\end{array}$ & & $\begin{array}{l}18 \\
(10.17 \%)\end{array}$ & \\
\hline Not at all important & $\begin{array}{l}25 \\
(0.87 \%)\end{array}$ & $2(0.63 \%)$ & $0(--)$ & $3(0.74 \%)$ & $\begin{array}{l}20 \\
(0.99 \%)\end{array}$ & & $2(1.13 \%)$ & \\
\hline TOTAL & $\begin{array}{l}2866 \\
(100 \%)\end{array}$ & $318(100 \%)$ & 125 (100\%) & 408 (100\%) & $\begin{array}{l}2015 \\
(100 \%)\end{array}$ & & $\begin{array}{l}177 \\
(100 \%\end{array}$ & \\
\hline $\begin{array}{l}\text { How much importance is given to talents/ } \\
\text { accomplishments when recruiting applicants for jobs } \\
\text { and/or positions in science and medicine? }\end{array}$ & & & & & & 0.0405 & & $<.0001$ \\
\hline
\end{tabular}


Table 5 Perceptions of research feasibility and the importance of mentoring (Continued)

\begin{tabular}{|c|c|c|c|c|c|c|c|}
\hline & $\begin{array}{l}\text { Total, } \\
\text { n (\%) }\end{array}$ & $\begin{array}{l}\text { Asian or } \\
\text { Pacific } \\
\text { Islander, } \mathrm{n} \\
\text { (\%) }\end{array}$ & $\begin{array}{l}\text { Black or } \\
\text { African } \\
\text { American, } \\
\text { n (\%) }\end{array}$ & $\begin{array}{l}\text { Multiracial } \\
\text { or Other, } \mathrm{n} \\
\text { (\%) }\end{array}$ & $\begin{array}{ll}\text { White, } & P \text { - } \\
\text { n (\%) } & \text { value }\end{array}$ & $\begin{array}{l}\text { Hispanic, } \\
\text { n (\%) }\end{array}$ & $\begin{array}{l}\text { Hispanic } \\
P \text {-value }\end{array}$ \\
\hline A great deal of importance & $\begin{array}{l}1170 \\
(30.9 \%)\end{array}$ & $152(37.4 \%)$ & $49(32.0 \%)$ & $174(32.5 \%)$ & $\begin{array}{l}795 \\
(29.5 \%)\end{array}$ & $70(30.2 \%)$ & \\
\hline A lot of importance & $\begin{array}{l}1835 \\
(48.5 \%)\end{array}$ & $181(44.5 \%)$ & $66(43.1 \%)$ & $256(47.9 \%)$ & $\begin{array}{l}1332 \\
(49.5 \%)\end{array}$ & $\begin{array}{l}110 \\
(47.4 \%)\end{array}$ & \\
\hline Moderate amount of importance & $\begin{array}{l}719 \\
(19.0 \%)\end{array}$ & $70(17.2 \%)$ & $36(23.5 \%)$ & $95(17.8 \%)$ & $\begin{array}{l}518 \\
(19.3 \%)\end{array}$ & $46(19.8 \%)$ & \\
\hline Little importance & $\begin{array}{l}60 \\
(1.58 \%)\end{array}$ & $3(0.74 \%)$ & $2(1.31 \%)$ & $9(1.68 \%)$ & $\begin{array}{l}46 \\
(1.71 \%)\end{array}$ & $3(1.29 \%)$ & \\
\hline None at all & $\begin{array}{l}2 \\
(0.05 \%)\end{array}$ & $1(0.25 \%)$ & $0(--)$ & $1(0.19 \%)$ & $0(--)$ & $3(1.29 \%)$ & \\
\hline TOTAL & $\begin{array}{l}3786 \\
(100 \%)\end{array}$ & $407(100 \%)$ & $153(100 \%)$ & $535(100 \%)$ & $\begin{array}{l}2691 \\
(100 \%)\end{array}$ & $\begin{array}{l}232 \\
(100 \%)\end{array}$ & \\
\hline $\begin{array}{l}\text { How much importance is given to connections/ } \\
\text { networking when recruiting applicants for jobs and/or } \\
\text { positions in science and medicine? }\end{array}$ & & & & & & & 0.0053 \\
\hline A great deal of importance & $\begin{array}{l}1223 \\
(32.3 \%)\end{array}$ & $152(37.3 \%)$ & $67(44.1 \%)$ & $213(39.8 \%)$ & $\begin{array}{l}791 \\
(29.4 \%)\end{array}$ & $\begin{array}{l}101 \\
(43.2 \%)\end{array}$ & \\
\hline A lot of importance & $\begin{array}{l}1603 \\
(42.3 \%)\end{array}$ & $157(38.5 \%)$ & $54(35.5 \%)$ & $205(38.3 \%)$ & $\begin{array}{l}1187 \\
(44.0 \%)\end{array}$ & $87(37.2 \%)$ & \\
\hline Moderate amount of importance & $\begin{array}{l}844 \\
(22.3 \%)\end{array}$ & $86(21.1 \%)$ & $27(17.8 \%)$ & $96(17.9 \%)$ & $\begin{array}{l}635 \\
(23.6 \%)\end{array}$ & $43(18.4 \%)$ & \\
\hline Little importance & $\begin{array}{l}118 \\
(3.11 \%)\end{array}$ & $12(2.94 \%)$ & $4(2.63 \%)$ & $21(3.93 \%)$ & $\begin{array}{l}81 \\
(3.01 \%)\end{array}$ & $3(1.28 \%)$ & \\
\hline TOTAL & $\begin{array}{l}3790 \\
(100 \%)\end{array}$ & $408(100 \%)$ & $152(100 \%)$ & $535(100 \%)$ & $\begin{array}{l}2695 \\
(100 \%)\end{array}$ & $\begin{array}{l}234 \\
(100 \%)\end{array}$ & \\
\hline
\end{tabular}

being not very or at all important (Black/African American 2.4\%; Multiracial/Other 8.2\%; White 12.1\%; Hispanic 11.3\%) (Table 5).

More Asian trainees (81.9\%) believed talents and accomplishments were given a great deal or a lot of importance in recruiting for jobs and positions in medicine compared to other groups (Black/African American 75.1\%; Multiracial/Other 80.4\%; White 79\%; Hispanic $77.6 \%)$. Hispanic (80.4\%) trainees were most likely to believe that connections and networking have a great deal of importance or a lot of importance when recruiting for jobs or positions in science and medicine, followed by Black/African American (79.6\%), Multiracial/Other (78.1\%), Asian (75.8\%), and White (73.4\%) trainees.

\section{Discussion}

Previous studies investigating demographic disparities in the physician-scientist workforce have primarily focused on identifying disparate trends in the prevalence of underrepresented minorities in medical school/physicianscientist training program admissions, representation among academic faculty, or research grant application or funding success rates. While characterizing these statistical trends is important, efforts to improve demographic representation in the physician-scientist workforce would likely benefit from an understanding of the unique perspectives and challenges experienced by URMs early in training, such that these trainees can be effectively mentored throughout their training and career. In this regard, the present study represents the first in-depth survey of a nationally representative cohort of pre-doctoral physician-scientist trainees to evaluate differences in career aspirations, perceptions, and obstacles between those who identify as URM and their non-URM peers. Our results identify several differences in the training experience, interests, and intended career trajectory between URM and non-URM trainees, which may help to inform URM trainee recruitment and mentorship efforts to improve diversity in the physicianscientist workforce.

\section{Academic and research interests}

One of the central goals of the study was to determine whether academic and research career interests vary according to trainee race/ethnic group. While we found that all racial/ethnic groups expressed a strong interest in pursuing a career in academia, the rates of academic interest did vary between groups, with Black/African American trainees reporting the lowest rates of interest in academia (40.8\%). Importantly, racial/ethnic 
differences in academic career interests persisted after controlling for several demographic and trainee characteristics, including sex, age, training stage, access to mentors, specialty intention, and method of medical school funding by multivariate regression modeling. In particular, while there were no differences in academic career interests between Asian and White trainee cohorts, Asian trainees and White trainees were each independently significantly more likely than Black/American trainees to report interest in academic careers. In addition, White trainees were significantly more likely than Multiracial/Other trainees to express an interest in academic careers. These disparities in academic career interests at the predoctoral level are consistent with recent studies showing declining trends in URM representation among academic medicine faculty across various clinical specialties $[17,18]$.

With respect to research interests, our data suggest that there exist racial/ethnic differences in research career intentions. Among non-URM trainees, Asians demonstrated the strongest interest in basic/translational research (19.1\%), while only $15.8 \%$ of White trainees shared this interest. Among URM trainees, there was a similar level of heterogeneity. While Black/African American respondents were least interested in basic/ translational research (14.3\%), Multiracial/Other (17.9\%) and Hispanic (17.0\%) trainees did express a substantial level of interest in this type of research. Similar to academic career interest, we found that after adjusting for multiple demographic and trainee covariates, Black/African American trainees remained significantly less likely than Asian and White trainees to report interest in basic research. However, it is interesting to note that Black/ African American trainees did express a stronger interest in clinical research $(8.4 \%)$ than other groups. These findings are important because previous studies have shown that participation in research during graduate medical training is significantly associated with full-time academic faculty appointment for URM trainees [19]. Together, this suggests that efforts to improve physician-scientist workforce diversity should include support and opportunities for URM physician-scientist trainees to engage in a broader range of research types from preclinical basic/translational research at the bench to patient centered research at the bedside.

\section{Value of professional activities: advocacy and international work}

URM trainees were more likely to express an interest in incorporating advocacy and international work into their careers. This may reflect a desire by URM trainees to give back to their community, as seen in the high proportion of URM faculty who report working in areas that address health disparities [20]. However, this early desire for advocacy may also represent a source of burnout and dissatisfaction at the clinical or research faculty stage. Advocacy work may be transformed by what some have called the "minority tax" [21], or the additional burdens placed on URM faculty, from a career aspiration into an obligation which promotes attrition [22]. Though independent longitudinal validation of general URM interest in advocacy work is required, consideration of these activities in the academic promotion and tenure process could incentivize URM trainees with these interests to continue along this career trajectory and incorporate such work into an academic career.

\section{Training and career obstacles}

The results from this survey identify key obstacles facing URM trainees at the predoctoral level. Concerning mechanisms for financing medical school, all racial/ethnic groups in this survey reported loans as the primary means of paying for medical school tuition. However, following loans, there were striking differences between trainees in different racial/ethnic groups. Whereas $24.3 \%$ of Asian, $23.3 \%$ of Multiracial/Other, and $13.5 \%$ of White trainees were dependent upon on family/partner support, only $2.5 \%$ of Black/African American and 5.8\% of Hispanic trainees reported this as a primary means of paying for medical school. The limited familial/partner financial support among Black/African American and Hispanic trainees is consistent with previous reports demonstrating that these groups carry and anticipate substantially more educational debt than their peers [23, 24]. These financial concerns have also been shown to extend to the undergraduate level, underscoring the compounding nature of financial debt in these demographic groups prior to beginning medical school training $[25,26]$. It has been suggested that perceived obstacles related to debt may subsequently influence specialty and career trajectories of these trainees, biasing trainees toward specialties and career practices with greater salaries and reimbursements [24, 27-30]. Although partial or full tuition scholarships are frequently offered by dual-degree programs, single-degree URM trainees interested in pursuing additional research training will likely disproportionately experience additional financial obstacles. The NIH Loan Repayment program (LRP) or other comparable funding mechanism could be leveraged to reduce this excess burden and encourage academic and research careers for single-degree URM physician-scientists [3, 31].

In addition to financial obstacles, perhaps most disturbingly, our survey demonstrates that Black/African American trainees reported experiencing significantly higher rates of discrimination and bias. These values ranged from 1.9 to 3.4-fold greater than trainees from other racial/ethnic groups. Several reports over the past 
few decades have noted that URM physicians report discrimination at significantly higher levels than White peers [32-34]. It is therefore disappointing and alarming that URM pre-doctoral trainees in this survey also reported experiencing such high rates of self-reported discrimination and bias at this stage in training, which is consistent with findings from other studies $[35,36]$. These results are all the more concerning when placed against the urgent need to diversify the academic medical and physician-scientist workforce [37]. Medical school faculty frequently associate discrimination with low career satisfaction, and one factor behind the limited number of URMs in the physicianscientist workforce may be discrimination in the earliest phases of training [32].

\section{Mentorship}

When exploring employment or other opportunities, non-URM trainees appeared to place more weight on talents and accomplishments, whereas URM trainees seemed to emphasize networking and connections. While URM trainees are more likely to have identified mentors, respondents did not indicate the nature of mentorship or the types of mentors (e.g. physicianscientists vs physicians), which could influence career choices. In this analysis, we also show that Black/African American trainees are less likely than trainees of other racial/ethnic groups to intend careers in academia and basic research. Owing to the extensive duration of training and the unique professional challenges associated with careers in academic medicine and basic research, perhaps one interpretation of these trends is that they reflect the dearth of available and accessible mentors in these career areas. Institutions and organizations aiming to improve diversity in physician-scientist training pipelines would do well to assign significant value to mentors and mentorship time for all trainees, including those who identify as URM.

\section{Limitations}

This study has several limitations. First, the survey response rate was $27 \%$, which is considerably lower than other physician-scientist trainee surveys conducted by single institutions or organizations such as the AAMC $[38,39]$. One potential explanation for the relatively low response rate in this analysis is that we utilized an extensive 70-question survey to comprehensively assess trainee attitudes and perceptions. A second limitation is that the proportion of respondents identifying as Asian or Pacific Islanders in our survey was about half that expected from AAMC data, most likely reflecting oversampling of White and multiracial or other populations, as these both exceeded the expected proportions based on AAMC data. Another important limitation of this study is its cross-sectional nature, which cannot rule out the possibility of discordance between trainee survey responses and their ultimate career destinations. However, other studies have suggested that physician-scientist trainees tend to pursue careers in accordance with their training [40]. Finally, although this analysis identifies many pertinent factors and obstacles associated with URM trainee career goals, it does not provide insight into the reasons for why these factors exist among URM trainees. One important direction for future studies on this topic is to specifically understand why the career factors and obstacles identified here are uniquely prevalent in URM trainee cohorts. Such analyses are critical for optimizing policy and advocacy efforts aimed at recruiting and retaining URM trainees into the physician-scientist pipeline.

\section{Conclusions}

A diverse physician-scientist workforce is critical for the future of academic biomedical research. Understanding the perspectives, experiences, and obstacles of URM physician-scientist trainees at the predoctoral level is an important step in efforts to diversify the physicianscientist workforce. In this cross-sectional analysis of over 4000 predoctoral physician-scientist trainees, we found that URM trainees do generally demonstrate an interest in pursuing academic and research activities in their career practices. However, URM trainees do differ from non-URM trainees in some career and specialty interests and in the importance and role of mentorship during training. These findings may serve to improve efforts to recruit and mentor the next generation of URM physician-scientists.

\section{Supplementary Information}

The online version contains supplementary material available at https://doi. org/10.1186/s12909-020-02328-6.

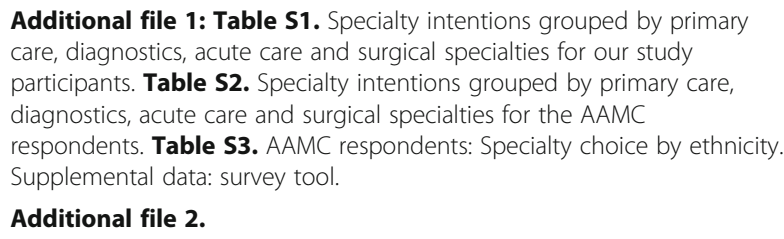

Additional file 1: Table S1. Specialty intentions grouped by primary care, diagnostics, acute care and surgical specialties for our study participants. Table S2. Specialty intentions grouped by primary care, diagnostics, acute care and surgical specialties for the AAMC respondents. Table S3. AAMC respondents: Specialty choice by ethnicity. Supplemental data: survey tool.

Additional file 2 .

\section{Acknowledgements}

The authors thank the American Physician Scientists Association leadership and staff for administrative and logistical support for this project. We thank the American Women's Medical Association for their help with survey distribution to their members. The authors thank Drs. Skip Brass, Laina King and Andrew Harrison for their thoughtful comments and review of this manuscript. We also thank the NIH R13 grant for helping to bring together a diverse trainee population that has helped to facilitate garnering response rates for the survey. Finally, we thank the AAMC for their help with obtaining the AAMC data used in this study for comparisons.

\section{Authors' contributions}

JMK and DD were involved with the study design, receiving IRB approval, and collecting participant responses. AS, CC, OT, HK, JMK participated in data 
analysis and interpretation of results. All authors contributed to drafting the manuscript and revising it for important intellectual content. All authors approve of the final version of the manuscript to be published.

\section{Funding}

This project received financial support from the University of Illinois at Chicago Center for Clinical and Translational Science grant UL1TR002003, from the American Physician Scientists Association, the Trustees' Council of Penn Women at the University of Pennsylvania, and the Burroughs Wellcome Fund. Authors were supported by fellowships from NIDDK (F31 DK108658-01) (ALS), NHLBI (F30 HL-126324) (AJA), NHLBI (F30 HL-154324) (OT). NHBLI T32 grant 5T32HL072742-10 to JMK.

\section{Availability of data and materials}

In accordance with IRB stipulations, data will not be posted online but can be made available at request.

\section{Ethics approval and consent to participate}

The study was reviewed and exempted by the Institutional Review Boards at the University of Illinois at Chicago and the University of Pennsylvania. All subjects consented to participate in this study.

\section{Consent for publication}

The IRB for this study was granted exempt status. In it, participants were asked to provide consent for possible publication of results, which all gave in order to proceed with the survey. All data in the present analysis were gathered from participants who consented for direct responses to be used for publication. Consent for direct quotes from their interviews to be published in this manuscript was not explicitly obtained and therefore, we do not have any direct quotes featured in the survey results.

\section{Competing interests}

The authors declare that they have no competing interests.

\section{Author details}

${ }^{1}$ Northwestern University, Chicago, USA. ${ }^{2}$ University of Pittsburgh, Pittsburgh, USA. ${ }^{3}$ Keck School of Medicine of USC, University of Southern California, Los Angeles, USA. ${ }^{4}$ Department of Internal Medicine, University of Washington, Seattle, WA, USA. ${ }^{5}$ University of Illinois, Chicago, IL, USA. ${ }^{6}$ Massachusetts General Hospital, Boston, USA. ${ }^{7}$ Section of Cardiovascular Medicine, Yale University School of Medicine, New Haven, CT 06511, USA.

\section{Received: 23 June 2020 Accepted: 21 October 2020}

\section{Published online: 11 November 2020}

\section{References}

1. National Institutes of Health: Notice of $\mathrm{NIH}^{\prime}$ Interest in Diversity Notice Number NOT-OD-20-031. https://grants.nih.gov/grants/guide/notice-files/ NOT-OD-20-031.html (2019) Accessed 10 Aug 2020.

2. Association of American Medical Colleges: Underrepresented in Medicine Definition. https://www.aamc.org/what-we-do/mission-areas/diversityinclusion/underrepresented-in-medicine (2003). Accessed 10 Aug 2020.

3. National Institutes of Health: Physician-scientist workforce (PSW) report. http://report.nih.gov/workforce/psw/index.aspx (2014). Accessed 19 Aug 2020.

4. Beech BM, Calles-Escandon J, Hairston KG, Langdon SE, Latham-Sadler BA, Bell RA. Mentoring programs for underrepresented minority faculty in academic medical centers: a systematic review of the literature. Acad Med. 2013;88(4):541-9.

5. Daley S, Wingard DL, Reznik V. Improving the retention of underrepresented minority Faculty in Academic Medicine. J Natl Med Assoc. 2006;98(9):143540.

6. Kosoko-Lasaki O, Sonnino RE, Voytko ML. Mentoring for women and underrepresented minority faculty and students: experience at two institutions of higher education. J Natl Med Assoc. 2006;98(9):1449-59.

7. Gotian R, Raymore JC, Rhooms SK, Liberman L, Andersen OS. Gateways to the laboratory: how an MD-PhD program increased the number of minority physician-scientists. Acad Med. 2017;92(5):628-34.

8. Fang D, Moy E, Colburn L, Hurley J. Racial and ethnic disparities in faculty promotion in academic medicine. JAMA. 2000;284(9):1085-92.
9. Andriole DA, Jeffe DB. Predictors of full-time faculty appointment among MD-PhD program graduates: a national cohort study. Med Educ Online. 2016;21(1):30941.

10. Palepu A, Carr PL, Friedman RH, Amos H, Ash AS, Moskowitz MA. Minority faculty and academic rank in medicine. JAMA. 1998;280(9):767-71.

11. The American Physician Scientists Association. https://www. physicianscientists.org (2003). Accessed 19 Aug 2020.

12. Kwan JM, Daye D, Schmidt ML, et al. Exploring intentions of physicianscientist trainees: factors influencing $\mathrm{MD}$ and $\mathrm{MD} / \mathrm{PhD}$ interest in research careers. BMC Med Educ. 2017;17(1):115.

13. Kwan JM, Toubat O, Harrison AM, et al. A Nationwide assessment of perceptions of research-intense academic careers among Predoctoral MD and MD-PhD trainees. J Clin Transl Sci. 2020;4(4):307-16.

14. National Institutes of Health: Research Training and Career Development. https://researchtraining.nih.gov/resources/faq (2015). Accessed 5 Mar 2020.

15. Association of American Medical Colleges: Enrollment, Graduates, and MD PhD Data. https://www.aamc.org/data-reports/students-residents/ interactive-data/2019-facts-enrollment-graduates-and-md-phd-data (2019). Accessed 21 Mar 2020.

16. Quinn KA. Graduate and professional student socialization regarding work and family in higher education. Ithaca: University of Washington; 2006.

17. Lett LA, Orji WU, Sebro R. Declining racial and ethnic representation in clinical academic medicine: a longitudinal study of 16 US medical specialties. PLoS One. 2018;13(11):e0207274.

18. Valenzuela F, Romero Arenas MA. Underrepresented in surgery: (lack of) diversity in academic surgery faculty. J Surg Res. 2020;254:170-4.

19. Jeffe DB, Yan $Y$, Andriole DA. Do research activities during college, medical school, and residency mediate racial/ethnic disparities in full-time faculty appointments at US medical schools? Acad Med. 2012;87(11):1582-93.

20. Pololi LH, Evans AT, Gibbs BK, Krupat E, Brennan RT, Civian JT. The experience of minority faculty who are underrepresented in medicine, at 26 representative U.S. medical schools. Acad Med. 2013;88(9):1308-14.

21. Rodriguez JE, Campbell KM, Pololi LH. Addressing disparities in academic medicine: what of the minority tax? BMC Med Educ. 2015;15:6.

22. Pololi L, Cooper LA, Carr P. Race, disadvantage and faculty experiences in academic medicine. J Gen Intern Med. 2010;25(12):1363-9.

23. Dugger RA, El-Sayed AM, Dogra A, et al. The color of debt: racial disparities in anticipated medical student debt in the United States. PLoS One. 2013;8(9):e74693.

24. Rosenblatt RA, Andrilla CHA. The impact of US medical students' debt on their choice of primary care careers: an analysis of data from the 2002 medical school graduation questionnaire. Acad Med. 2005;80(9):815-9.

25. U.S. Department of Education National Center for Education Statistics: National Postsecondary Student Aid Study. https://nces.ed.gov/pubsearch/ pubsinfo.asp?pubid=2013165 (2013). Accessed 28 Mar 2020.

26. Association of American Medical Colleges: Diversity in Medicine Facts and Figures. https://www.aamc.org/data-reports/workforce/interactive-data/ figure-10-amount-premedical-education-debt-us-medical-schoolmatriculants-race/ethnicity-academic (2019). Accessed 19 Aug 2020.

27. Grayson MS, Newton DA, Thompson LF. Payback time: the associations of debt and income with medical student career choice. Med Educ. 2012;46(10):983-91

28. Andriole DA, Whelan AJ, Jeffe DB. Characteristics and career intentions of the emerging MD/PhD workforce. JAMA. 2008;300(10):1165-73.

29. Garcia AN, Kuo T, Arangua L, et al. Factors associated with medical school graduates' intention to work with underserved populations: policy implications for advancing workforce diversity. Acad Med. 2018;93(1):82-9.

30. Pisaniello MS, Asahina AT, Bacchi S, et al. Effect of medical student debt on mental health, academic performance, and specialty choice: a systematic review. BMJ Open. 2019;9(7):e029980.

31. Ley TJ, Rosenberg LE. The physician-scientist career pipeline in 2005. JAMA. 2005;294(11):1343-51.

32. Peterson NB, Friedman RH, Ash AS, Franco S, Carr PL. Faculty self-reported experience with racial and ethnic discrimination in academic medicine. J Gen Intern Med. 2004;19:259-65.

33. Nunez-Smith M, Pilgrim N, Wynia M, et al. Race/ethnicity and workplace discrimination: results of a national survey of physicians. J Gen Intern Med. 2009:24(11):1198-204.

34. Filut A, Alvarez M, Carnes M. Discrimination toward physicians of color: a systematic review. J Natl Med Assoc. 2020;112(2):117-40.

35. Fnais $\mathrm{N}$, Soobiah $\mathrm{C}$, Chen $\mathrm{MH}$, et al. Harassment and discrimination in medical training: a systematic review and meta-analysis. Acad Med. 2014; 89(5):817-27. 
36. Hill KA, Samuels EA, Gross CP, et al. Assessment of the prevalence of medical student mistreatment by sex, race/ethnicity, and sexual orientation. JAMA. 2020;180(5):653-65.

37. Milewicz DM, Lorenz RG, Dermody TS, et al. Rescuing the physician-scientist workforce: the time for action is now. J Clin Invest. 2015;125(10):3742-7.

38. Jeffe $\mathrm{DB}$, Andriole DA, Wathington $\mathrm{HD}$, et al. Educational outcomes for students enrolled in MD-PhD programs at medical school matriculation, 1995-2000: a national cohort study. Acad Med. 2014;89(1):84-93.

39. Jeffe $\mathrm{DB}$, Andriole $\mathrm{DA}$, Wathington $H \mathrm{HD}$, et al. The emerging physicianscientist workforce: demographic, experiential, and attitudinal predictors of MD-PhD program enrollment. Acad Med. 2014;89(10):1398-407.

40. Brass LF, Akabas MH, Burnley LD, et al. Are MD-PhD programs meeting their goals? An analysis of career choices made by graduates of $24 \mathrm{MD}-\mathrm{PhD}$ programs. Acad Med. 2010;85(4):692-701.

\section{Publisher's Note}

Springer Nature remains neutral with regard to jurisdictional claims in published maps and institutional affiliations.

Ready to submit your research? Choose BMC and benefit from:

- fast, convenient online submission

- thorough peer review by experienced researchers in your field

- rapid publication on acceptance

- support for research data, including large and complex data types

- gold Open Access which fosters wider collaboration and increased citations

- maximum visibility for your research: over $100 \mathrm{M}$ website views per year

At BMC, research is always in progress.

Learn more biomedcentral.com/submissions 